\title{
LOGICS OF INVOLUTIVE STONE ALGEBRAS
}

\author{
SÉRGIO MARCELINO \\ SQIG - Instituto de Telecomunicações, Portugal \\ Departamento de Matemática, Instituto Superior Técnico, Universidade de Lisboa
}

UMBERTO RIVIECCIO*

Departamento de Informática e Matemática Aplicada, Universidade Federal do Rio Grande do Norte, Natal (RN), Brasil

\begin{abstract}
An involutive Stone algebra (IS-algebra) is a structure that is simultaneously a De Morgan algebra and a Stone algebra (i.e. a pseudo-complemented distributive lattice satisfying the well-known Stone identity, $\sim x \vee \sim \sim x \approx 1$ ). IS-algebras have been studied algebraically and topologically since the 1980's, but a corresponding logic (here denoted $\mathcal{I} \mathcal{S}_{\leq}$) has been introduced only very recently. The logic $\mathcal{I} \mathcal{S}_{\leq}$is the departing point for the present study, which we then extend to a wide family of previously unknown logics defined from IS-algebras. We show that $\mathcal{I} \mathcal{S}_{\leq}$is a conservative expansion of the Belnap-Dunn four-valued logic (i.e. the order-preserving logic of the variety of De Morgan algebras), and we give a finite Hilbert-style axiomatization for it. More generally, we introduce a method for expanding conservatively every super-Belnap logic so as to obtain an extension of $\mathcal{I} \mathcal{S}_{\leq}$. We show that every logic thus defined can be axiomatized by adding a fixed finite set of rule schemata to the corresponding superBelnap base logic. We also consider a few sample extensions of $\mathcal{I} \mathcal{S}_{\leq}$that cannot be obtained in the above-described way, but can nevertheless be axiomatized finitely by other methods. Most of our axiomatization results are obtained in two steps: through a multiple-conclusion calculus first, which we then reduce to a traditional one. The multiple-conclusion axiomatizations introduced in this process, being analytic, are of independent interest from a proof-theoretic standpoint. Our results entail that the lattice of super-Belnap logics (which is known to be uncountable) embeds into the lattice of extensions of $\mathcal{I} \mathcal{S}_{\leq}$. Indeed, as in the super-Belnap case, we establish that the finitary extensions of $\mathcal{I} \mathcal{S}_{\leq}$are already uncountably many.
\end{abstract}

\section{INTRODUCTION}

Involutive Stone algebras (from now on, IS-algebras) were first considered in the papers 8 9 within a study of finite-valued Łukasiewicz logics and, more specifically, in connection with the algebraic structures nowadays known as Łukasiewicz-Moisil algebras. The term 'involutive' is due to the observation that every IS-algebra has a primitive negation operation $\sim$ that satisfies the involutive law $(\sim \sim x \approx x)$, whereas 'Stone' refers to the existence of a term-definable pseudo-complement operation $\neg$ that satisfies the well-known Stone identity

E-mail addresses: smarcel@math.tecnico.ulisboa.pt, urivieccio@dimap.ufrn.br, phone: +558432153814. Date: March 15, 2021. 
$\neg x \vee \neg \neg x \approx 1$. From an algebraic point of view, IS-algebras are a variety of De Morgan algebras endowed with an additional unary operation (here denoted by $\nabla$ ); alternatively, ISalgebras can be viewed as the subclass of De Morgan algebras that satisfy certain structural properties ensuring the definability of $\nabla$. The structural connection between De Morgan and IS-algebras will indeed play a prominent role in the present paper.

De Morgan algebras form a variety that is well known in non-classical logic as the algebraic counterpart of $\mathcal{B}$, the four-valued Belnap-Dunn logic 2, 14]. From a logical point of view, $\mathcal{B}$ can be viewed as a weakening of classical two-valued logic designed to allow for both paraconsistency (in that $\mathcal{B}$ does not validate the rule $p \wedge \sim p \vdash q$, known as ex contradictione quodlibet) and paracompleteness (in that $\mathcal{B}$ does not validate the principle of excluded middle, $\vdash p \vee \sim p)$. De Morgan algebras can thus be viewed as a generalization of a Boolean algebras on which the operation $\sim$ (that interprets the negation connective) need not be a Boolean complement, i.e. the classical laws $x \wedge \sim x \approx 0$ and $x \vee \sim x \approx 1$ need not be satisfied. The involutive and the De Morgan laws are however valid on every De Morgan algebra (see Definition 3.1).

As mentioned above, involutive Stone algebras may be regarded as a subclass of De Morgan algebras characterized by certain structural properties. From this perspective, an involutive Stone algebra may be viewed as a De Morgan algebra $\mathbf{A}$ having an additional unary operation $\nabla$ that receives an arbitrary element $a \in A$ as argument and outputs a certain 'classical' element $\nabla a$ (i.e. an element that possesses a Boolean complement in A). Just as not every distributive lattice can be equipped with a Boolean complement operation, so not every De Morgan algebra can be endowed with an operation $\nabla$ meeting the above requirement. However, if such an operation is definable, then it is unique.

One might say that, on every De Morgan algebra $\mathbf{A}$, the behaviour of $\nabla$ provides a measure of how far $\mathbf{A}$ is from being Boolean: the limit cases being, at one end of the spectrum, Boolean algebras themselves (on which $\nabla$ is the identity map) and, at the other, the algebras (such as those depicted in Figure 22) where the only Boolean elements are the top and the bottom. These structural requirements on $\nabla$ can be completely captured by means of identities [9, Thm. 2.1]. Therefore, regardless of the preceding considerations, IS-algebras can be simply introduced as a variety of De Morgan algebras having an extra unary operation $\nabla$ that is required to satisfy four additional identities (see Definition 3.2).

A logic associated to IS-algebras has been considered for the first time in [6, 7]. In the present paper, we shall denote this logic by $\mathcal{I S}_{\leq}$, suggesting that $\mathcal{I S}_{\leq}$is the orderpreserving logic canonically associated to the variety of IS-algebras (see Section 2 for the relevant definitions). As we will show, $\mathcal{I} \mathcal{S}_{\leq}$is a conservative expansion of the Belnap-Dunn $\operatorname{logic} \mathcal{B}$, which is itself the order-preserving logic of the variety of De Morgan lattices. We are moreover going to prove that, between the logics extending $\mathcal{B}$ (known as super-Belnap logics since the paper [21]) and the extensions of $\mathcal{I S}_{\leq}$, a connection can be established and exploited in order to obtain a number of non-trivial results.

The background facts we shall need on the Belnap-Dunn logic can be found in [14, including a complete Hilbert-style axiomatization and a characterization of the reduced matrix models (see Section 22). For further information on super-Belnap logics, we refer the reader to the papers 21, 1, 20, from which we shall also import a few results as needed.

The rest of the paper is organized as follows. Section 2 introduces the generic algebraic and logical notions that will be used in the following ones. Section 3 contains the basic algebraic results on De Morgan and involutive Stone algebras. In Section 4 we look at the logic $\mathcal{I} \mathcal{S}_{\leq}$of involutive Stone algebras from a semantical point of view. We observe that $\mathcal{I S} \leq$ non-protoalgebraic (Proposition 4.2) and can be characterized by a single finite 
matrix (Proposition 4.1). We further introduce a simple operation on logical matrices that allows us to associate, to any given super-Belnap logic, a logic extending $\mathcal{I} \mathcal{S}_{\leq}$is such a way that the latter is a conservative expansion of the former (Lemma 4.11). This entails that the lattice of super-Belnap logics is embeddable into the lattice of extensions of $\mathcal{I} \mathcal{S}_{\leq}$ (Corollary 4.13), which in turn tells us that the latter must have at least the cardinality of the continuum (Corollary 4.14).

In Section 5 we present a uniform method of axiomatizing all the extensions of $\mathcal{I} \mathcal{S}_{<}$ that are defined from super-Belnap logics via the construction introduced in Section 4 (Corollary [5.7. Proposition [5.9); a complete axiomatization for $\mathcal{I S}_{\leq}$is thus obtained as a special application (Example 5.8). In order to achieve these results, we take a little detour through the realm of multiple-conclusion logics and the calculi that correspond to them. In Subsection 5.2 a number of logics extending $\mathcal{I} \mathcal{S}_{\leq}$(corresponding to substructures of the matrix that defines $\mathcal{I} \mathcal{S}_{\leq}$) are axiomatized by a uniform application of the general method; these include logics obtained by adding the $\nabla$ connective to well-known extensions of $\mathcal{B}$, such as G. Priest's Logic of Paradox and the strong and weak three-valued logics due to S. C. Kleene. In Subsection 5.3 we axiomatize a few extensions of $\mathcal{I} \mathcal{S}_{\leq}$are not obtained in this way from a super-Belnap logic, among which we find the three-valued Łukasiewicz(Moisil) logic. For the latter results we cannot apply the above-mentioned method, so we need to take a longer detour through multiple-conclusion logics and analytical calculi (Subsection [5.4). Finally, Section 6 contains a few concluding remarks and suggestions for future research.

\section{Algebraic And logical preliminaries}

In this Section we recall the main algebraic and logical notions that will be needed in the following ones. We assume familiarity with basic results of lattice theory [13], universal algebra [3] and the general theory of logical calculi [23, 16, 15].

We shall denote by $\mathbf{A}, \mathbf{B}$ etc. algebras over a given algebraic similarity type $\Sigma$. The set of $\Sigma$-homomorphisms between two algebras $\mathbf{A}$ and $\mathbf{B}$ will be denoted by $\operatorname{Hom}(\mathbf{A}, \mathbf{B})$. Given $\Sigma$-algebras $\mathbf{A}, \mathbf{B}$ and a sub-signature $\Sigma^{\prime} \subseteq \Sigma$, we denote by $\operatorname{Hom}_{\Sigma^{\prime}}(\mathbf{A}, \mathbf{B})$ the set of functions $h: A \rightarrow B$ that are only required to preserve the operations in $\Sigma^{\prime}$. The algebra of formulas over a signature $\Sigma$ will be denoted by $\mathbf{F m}_{\Sigma}$ (or simply by $\mathbf{F m}$, if $\Sigma$ is clear from the context), and its elements by $\varphi, \psi$ etc. Given a class $\mathrm{K}$ of similar algebras, we denote by $\mathbb{I}(\mathrm{K}), \mathbb{H}(\mathrm{K}), \mathbb{S}(\mathrm{K}), \mathbb{P}(\mathrm{K}), \mathbb{P}_{S}(\mathrm{~K})$ the classes formed by closing $\mathrm{K}$ under (respectively), isomorphisms, homomorphisms, subalgebras, direct products and subdirect products. A variety is a class $\mathrm{K}$ of algebras that is closed under $\mathbb{H}, \mathbb{S}, \mathbb{P}$, or, equivalently, that is definable by means of algebraic identities. A quasivariety is a class $\mathrm{K}$ of algebras that is definable by means of quasi-identities, that is, implications having a finite number of identities as premiss and a single identity as conclusion. The variety (resp. quasivariety) generated by $\mathrm{K}$ will be denoted by $\mathbb{V}(\mathrm{K})$ and $\mathbb{Q}(\mathrm{K})$. Every variety $\mathrm{V}$ is generated by the class $\mathrm{V}_{\text {si }}$ of its subdirectly irreducible members, defined as follows: an algebra $\mathbf{A}$ is subdirectly irreducible if $\mathbf{A}$ has a minimum congruence above the identity relation (as a special case, we say that $\mathbf{A}$ is simple if $\mathbf{A}$ has exactly two congruences). For every variety $\mathrm{V}$, we have $\mathrm{V}=\mathbb{V}\left(\mathrm{V}_{s i}\right)=\mathbb{P}_{S}\left(\mathrm{~V}_{s i}\right)$.

We view a (propositional, single-conclusion) logic as a structural consequence relation on $\wp(F m) \times F m$ (see e.g. [15, Def. 1.5]). The symbol $\vdash$ will be used to denote arbitrary logics.

We say that a logic $\vdash_{2}$ is an extension of $\vdash_{1}$ when both logics share the same propositional language $\Sigma$ and $\vdash_{1} \subseteq \vdash_{2}$. The family of all extensions of a logic $\vdash$ forms a complete lattice (in which the meet is the intersection); in this paper we shall be concerned with the lattice 
of extensions of the logic $\mathcal{I} \mathcal{S}_{\leq}$of involutive Stone algebras, and will relate it to the lattice of extensions of the Belnap-Dunn logic $\mathcal{B}$ (i.e. the super-Belnap logics). We say that a logic $\vdash_{2}$ over a language $\Sigma_{2}$ is an expansion of a logic $\vdash_{1}$ over $\Sigma_{1}$ when $\Sigma_{1} \subseteq \Sigma_{2}$ and $\vdash_{1} \subseteq \vdash_{2}$. We speak of a conservative expansion when both consequence relations coincide on the formulas over $\Sigma_{1}$.

A (logical) matrix is a pair $\mathbb{M}=\langle\mathbf{A}, D\rangle$ where $\mathbf{A}$ is an algebra and $D \subseteq A$ is a subset of designated elements. One defines the notions of isomorphism, homomorphism, submatrix and product of matrices as straightforward extensions of the corresponding universal algebraic constructions (see [23, 16] for details). Given a matrix $\mathbb{M}=\langle\mathbf{A}, D\rangle$ with $\mathbf{A}$ a $\Sigma$ algebra, we let $\operatorname{Val}(\mathbb{M})=\operatorname{Hom}\left(\mathbf{F m}_{\Sigma}, \mathbf{A}\right)$. We denote by Log the mapping that associates a logic to a class of matrices in the standard fashion. Indeed, each matrix determines a logic (denoted $\log \mathbb{M}$ or $\vdash_{\mathbb{M}}$ ) as follows: for all $\Gamma \cup\{\varphi\} \subseteq F m$, one lets $\Gamma \vdash_{\mathbb{M}} \varphi$ iff, for every valuation $v: \mathbf{F m} \rightarrow \mathbf{A}, v[\Gamma]=\{v(\gamma): \gamma \in \Gamma\} \subseteq D$ entails $v(\varphi) \in D$. To a class of matrices $\mathcal{M}=\left\{\mathbb{M}_{i}: i \in I\right\}$, we associate the $\operatorname{logic} \log \mathcal{M}=\vdash_{\mathcal{M}}:=\bigcap\left\{\vdash_{\mathbb{M}_{i}}: i \in I\right\}$. We say that a matrix $\mathbb{M}$ is a model of a logic $\vdash$ when $\vdash \subseteq \vdash_{\mathbb{M}}$, that is, when $\Gamma \vdash \varphi$ entails $\Gamma \vdash_{\mathbb{M}} \varphi$, for all $\Gamma \cup\{\varphi\} \subseteq F m$.

Every matrix $\mathbb{M}=\langle\mathbf{A}, D\rangle$ has an associated Leibniz congruence $\boldsymbol{\Omega}_{\mathbf{A}}(D)$, which is the greatest congruence on A that is compatible with $D$ in the following sense: for all $a \in D$ and $b \in A$, if $\langle a, b\rangle \in \Omega_{\mathbf{A}}(D)$, then $b \in D$. This property allows one to define the quotient matrix $\mathbb{M}^{*}=\left\langle\mathbf{A} / \boldsymbol{\Omega}_{\mathbf{A}}(D), D / \boldsymbol{\Omega}_{\mathbf{A}}(D)\right\rangle$, which is known as the reduction of $\mathbb{M}$. A matrix $\mathbb{M}$ is reduced if $\Omega_{\mathrm{A}}(D)$ is the identity relation, so no further reduction is possible. Reduced matrices are important in the study of algebraic models of logics, because, for every matrix $\mathbb{M}$, one has $\log \mathbb{M}=\log \mathbb{M}^{*}$. It follows that every logic coincides with the logic determined by the class of all its reduced matrix models. In algebraic logic, two classes of algebras, $\mathrm{Alg}^{*}(\vdash)$ and $\operatorname{Alg}(\vdash)$, are traditionally associated to a given logic $\vdash$. The former is defined as follows: $\operatorname{Alg}^{*}(\vdash):=\{\mathbf{A}$ : there is $D \subseteq A$ such that $\langle\mathbf{A}, D\rangle$ is a reduced matrix for $\vdash\}$ By the characterization of [16, Thm. 2.23], the latter class can be introduced as follows $\operatorname{Alg}(\vdash):=\mathbb{P}_{S}\left(\operatorname{Alg}^{*}(\vdash)\right)$.

Let $\mathrm{K}$ be a class of algebras such that each $\mathbf{A} \in \mathrm{K}$ has a semilattice reduct with top element 1. From $K$ one can obtain a finitary logic $\vdash_{\bar{K}}$ as follows. One lets $\emptyset \vdash_{\bar{K}} \varphi$ if and only if the identity $\varphi \approx 1$ is valid in $\mathrm{K}$ and, for all $\Gamma \cup\{\varphi\} \subseteq F m$ such that $\Gamma \neq \emptyset$, one lets $\Gamma \vdash_{\widehat{\mathrm{K}}}^{\leq} \varphi$ iff there are $\gamma_{1}, \ldots, \gamma_{n} \in \Gamma$ such that the identity $\gamma_{1} \wedge \ldots \wedge \gamma_{n} \wedge \varphi \approx \varphi$ is valid in $\mathrm{K}$. We shall call $\vdash_{\mathrm{K}}$ the order-preserving logic associated to $\mathrm{K}$. Observe that, by definition, $\mathrm{K}$ and $\mathbb{V}(\mathrm{K})$ define the same logic; thus, the order-preserving logics considered in the literature are usually associated to varieties of semilattice-based algebras. We note that, if $\mathrm{V}$ is a variety of algebras having a bounded distributive lattice reduct (as will always be the case in the present paper), then $\vdash_{\mathrm{V}}^{\leq}$coincides with the logic defined by the class of matrices $\{\langle\mathbf{A}, F\rangle: \mathbf{A} \in \mathrm{V}, F \subseteq A$ is a (non-empty) lattice filter of $\mathbf{A}\}$.

\section{De Morgan and involutive Stone algebras}

In this Section we recall the main definitions and basic results on the classes of algebras involved.

Definition 3.1. A De Morgan lattice is an algebra $\mathbf{A}=\langle A ; \wedge, \vee, \sim\rangle$ of type $\langle 2,2,1\rangle$ such that $\langle A ; \wedge, \vee\rangle$ is a distributive lattice and the following identities are satisfied:

(DM1) $\sim(x \vee y) \approx \sim x \wedge \sim y$.

(DM2) $\sim(x \wedge y) \approx \sim x \vee \sim y$.

(DM3) $x \approx \sim \sim x$. 
A De Morgan algebra is a De Morgan lattice whose lattice reduct is bounded (thus we include constant symbols $\perp$ and $T$ in the algebraic signature) and satisfies the following identities: $\sim \top \approx \perp$ and $\sim \perp \approx \top$.

Figure 1. (All the) subdirectly irreducible De Morgan algebras.

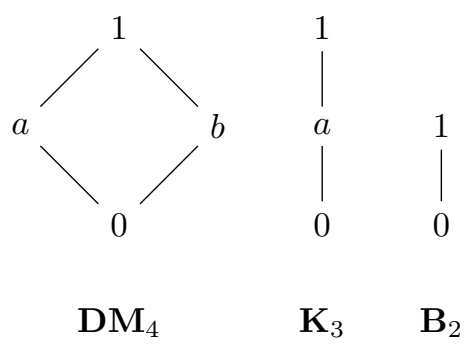

Figure1 depicts the (only) subdirectly irreducible De Morgan algebras. On each algebra, the lattice operations are determined by the diagram. The negation is defined on $\mathbf{D} \mathbf{M}_{4}$ by $\sim 0=1, \sim 1=0, \sim a=a$ and $\sim b=b$. These prescriptions apply to $\mathbf{K}_{3}$ and $\mathbf{B}_{2}$ as well viewed as subalgebras of $\mathbf{D M}_{4}$. Obviously $\mathbf{B}_{2}$ is the two-element Boolean algebra, and $\mathbf{K}_{3}$ is the three-element Kleene algebra associated to the three-valued logics originating from the work of S.C. Kleeneㄹ.

Definition 3.2. An involutive Stone algebra (IS-algebra) is an algebra $\mathbf{A}=\langle A ; \wedge, \vee, \sim, \nabla, 0,1\rangle$ of type $\langle 2,2,1,1,0,0\rangle$ such that $\langle A ; \wedge, \vee, \sim, \perp, \top\rangle$ is a De Morgan algebra and the following identities are satisfied:

(IS1) $\nabla \perp \approx \perp$.

(IS2) $x \approx x \wedge \nabla x$.

(IS3) $\nabla(x \wedge y) \approx \nabla x \wedge \nabla y$.

(IS4) $\sim \nabla x \wedge \nabla x \approx \perp$.

The class of IS-algebras will be denoted IS. The name 'involutive Stone algebras' is motivated by the following observation. For every IS-algebra $\mathbf{A}=\langle A ; \wedge, \vee, \sim, \nabla, \perp, \top\rangle$, the operation $\neg$ that realizes the term $\neg x:=\sim \nabla x$ is a pseudo-complement; moreover, $\mathbf{A}$ satisfies the so-called Stone identity $\neg x \vee \neg \neg x \approx T$. Hence, $\langle A ; \wedge, \vee, \neg, \perp, \top\rangle$ is a Stone algebra 2. Conversely, given an algebra $\langle A ; \wedge, \vee, \sim, \neg, \perp, \top\rangle$ that has both a De Morgan negation and a pseudo-complement operation, upon defining $\nabla x:=\neg \neg x$, one has that $\langle A ; \wedge, \vee, \sim, \nabla, 0,1\rangle$ is an IS-algebra if and only if the following identity is satisfied: $\neg x=$ $\sim \neg \neg x$ [9, Remark 2.2].

The variety of IS-algebras is generated by the six-element algebra $\mathbf{I S}_{6}$, which is shown in Figure 2 together with its subalgebras $\mathbf{I S}_{5}, \mathbf{I S}_{4}$ and $\mathbf{I S}_{3}$. Our notation reflects the observation that the De Morgan algebra reduct of $\mathbf{I S}_{6}$ is obtained by adjoining a new top $\hat{1}$ and a new bottom $\hat{0}$ element to the De Morgan algebra $\mathbf{D} \mathbf{M}_{4}$, and by extending the De Morgan operations in the obvious way (in particular, $\sim \hat{1}=\hat{0}$ and $\sim \hat{0}=\hat{1}$ ); cf. Proposition 3.4

\footnotetext{
${ }^{1}$ Formally, a Kleene lattice (algebra) is defined as a De Morgan lattice (algebra) that satisfies $x \wedge \sim x \leq$ $y \vee \sim y$. It is well known that the variety of Kleene lattices (algebras) is $\mathbb{V}\left(\mathbf{K}_{\mathbf{3}}\right)$.

${ }^{2}$ Formally, a Stone algebra can be defined as a bounded distributive lattice $\langle A ; \wedge, \vee, \neg, \perp, \top\rangle$ endowed by an extra unary operation $\neg$ that satisfies, for all $a, b \in A$, the following requirements: (i) $a \wedge b=0$ iff $a \leq \neg b$, and (ii) $\neg a \vee \neg \neg a=\top$.
} 
Figure 2. (All the) subdirectly irreducible IS-algebras.

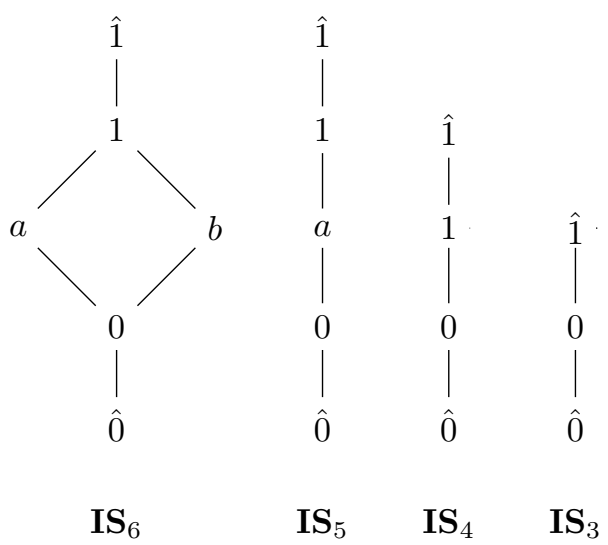

Likewise, $\mathbf{I S}_{5}$ is obtained from $\mathbf{K}_{\mathbf{3}}$, and $\mathbf{I} \mathbf{S}_{4}$ from $\mathbf{B}_{\mathbf{2}}$ (we can view $\mathbf{I S}_{3}$ as obtained in the same way if we start from the one-element trivial De Morgan algebra); this observation will be central in our approach to IS-algebras (see Section 4). The operation $\nabla$ is given on $\mathbf{I S}_{6}$ (and on its subalgebras) by $\nabla \hat{0}=\hat{0}$ and $\nabla 0=\nabla a=\nabla b=\nabla 1=\nabla \hat{1}=\hat{1}$. Observe that $\mathbf{I S}_{2}$ is isomorphic to the two-element Boolean algebra $\mathbf{B}_{\mathbf{2}}$ (on which $\nabla$ is the identity map). It is also well known that, upon defining $x \Rightarrow y:=(\nabla \sim x \vee y) \wedge(\nabla y \vee \sim x)$, the algebra IS $_{3}$ can be endowed with an MV-algebra structure [9, Thm. 2.9]. Conversely, on the three-element MV-algebra, one obtains an IS-algebra structure by letting $\nabla x:=\sim x \Rightarrow x$. Thus $\mathbf{I S}_{3}$ can be viewed as the three-element MV-algebra. On the other hand, on $\mathbf{I S}_{4}$ and $\mathbf{I S}_{5}$ the Lukasiewicz implication is not definable (to see this, it is sufficient to observe that neither of these algebras is simple, whereas it is known that every finite MV-chain is [10, Cor. 3.5.4]. The following result is an easy consequence of the observations in [9], and entails that, to verify the validity on all IS-algebras of not only identities but also quasi-identities, it is sufficient to test them on $\mathbf{I S}_{6}$.

Proposition 3.3. IS $=\mathbb{V}\left(\mathbf{I S}_{6}\right)=\mathbb{Q}\left(\mathbf{I S}_{6}\right)$.

Proof. That IS $=\mathbb{V}\left(\mathbf{I S}_{6}\right)$ is well-known [7, Cor. 3.5]. A sufficient condition for having $\mathbb{V}\left(\mathbf{I S}_{6}\right)=\mathbb{Q}\left(\mathbf{I S}_{6}\right)=\mathbb{I S P}\left(\mathbf{I S}_{6}\right)$ is that all the subdirectly irreducible algebras in $\mathbb{V}\left(\mathbf{I} \mathbf{S}_{6}\right)$ be subalgebras of $\mathbf{I S}_{6}$ (see e.g. [11, Thm. 3.6.ii]). The latter indeed holds, and has been observed in 9]; see Thm. 2.8 therein and the subsequent remarks.

The following easy observation will be very useful in our study of IS-logics from Section 4 on. Given a De Morgan algebra $\mathbf{A}$, let $\mathbf{A}^{\nabla}=\langle A \cup\{\hat{0}, \hat{1}\}, \nabla\rangle$ be the algebra defined as follows. The lattice reduct of $\mathbf{A}^{\nabla}$ is obtained by adjoining a new top element $\hat{1}$ and a new bottom element $\hat{0}$ to the lattice reduct of $\mathbf{A}$. The De Morgan negation $\sim$ is extended to $\mathbf{A}^{\nabla}$ in the obvious way, i.e. by letting $\sim \hat{1}=\hat{0}$ and $\sim \hat{0}=\hat{1}$. Furthermore, the unary operator $\nabla$ is defined as follows: $\nabla \hat{0}=\hat{0}$ and $\nabla a=\nabla \hat{1}=\hat{1}$ for all $a \in A$. It is then clear that the $\nabla$-free reduct of $\mathbf{A}^{\nabla}$ is a De Morgan algebra, and it is very easy to check that $\nabla$ satisfies all the properties required by Definition 3.1 .

The following easy observation will be very useful in our study of IS-logics from Section 4 on. Let $\mathbf{A}=\langle A ; \wedge, \vee, \sim, \perp, \top\rangle$ be an algebra in the language of De Morgan algebras. Given $\hat{0}, \hat{1} \notin A$, we define the algebra $\mathbf{A}^{\nabla}=\langle A \cup\{\hat{0}, \hat{1}\}, \nabla\rangle$ as follows: 


$$
\begin{aligned}
& \nabla x:=\left\{\begin{array}{ll}
\hat{0} \quad \text { if } x=\hat{0} \\
\hat{1} \quad \text { otherwise }
\end{array} \quad \sim x:= \begin{cases}\sim_{\mathbf{A}} x & \text { if } x \in A \\
\hat{0} & \text { if } x=\hat{1} \\
\hat{1} & \text { if } x=\hat{0}\end{cases} \right. \\
& x \wedge y:= \begin{cases}x \wedge_{\mathbf{A}} y & \text { if } x, y \in A \\
\hat{1} & \text { if } x=y=\hat{1} \\
\hat{0} & \text { if } x=\hat{0} \text { or } y=\hat{0} \\
z & \text { if }\{x, y\}=\{\hat{1}, z\}, z \in A\end{cases} \\
& \qquad \begin{array}{ll}
\top:=\hat{1} & \perp:=\hat{0} .
\end{array}
\end{aligned}
$$

In case $\mathbf{A}$ is a De Morgan algebra, it is clear that the $\nabla$-free reduct of $\mathbf{A}^{\nabla}$ is also a De Morgan algebra. Moreover, it is very easy to check that the above-defined $\nabla$ operation satisfies all the properties required by Definition 3.1. Thus, we have the following.

Proposition 3.4. For every De Morgan algebra $\mathbf{A}$, the above-defined algebra $\mathbf{A}^{\nabla}$ is an IS-algebra.

\section{Semantical CONSIDERAtions ON IS-LOGiCS}

It is shown in [7, Thm 5.2] the order-preserving logic of the variety IS (which we denote by $\mathcal{I} \mathcal{S}_{\leq}$) coincides with the logic determined by the closure system of all lattice filters on the generating algebra $\mathbf{I S}_{6}$ (which are the principal up-sets $\uparrow 0, \uparrow a, \uparrow b, \uparrow 1$, and $\{\hat{1}\}$ ). Since the matrices $\left\langle\mathbf{I S}_{6}, \uparrow a\right\rangle$ and $\left\langle\mathbf{I S}_{6}, \uparrow b\right\rangle$ define the same logic [7, Lemma 5.4], we have that $\mathcal{I} \mathcal{S}_{\leq}$ is determined by the following set of matrices: $\left\{\left\langle\mathbf{I S}_{6}, \uparrow 0\right\rangle,\left\langle\mathbf{I S}_{6}, \uparrow a\right\rangle,\left\langle\mathbf{I S}_{6}, \uparrow 1\right\rangle,\left\langle\mathbf{I S}_{6},\{\hat{1}\}\right\rangle\right\}$. This result can be further sharpened, as the following Proposition shows.

Proposition 4.1. $\mathcal{I} \mathcal{S}_{\leq}=\log \left\langle\mathbf{I S}_{6}, \uparrow a\right\rangle$.

Proof. Observe that the matrices $\left\langle\mathbf{I S}_{6}, \uparrow 1\right\rangle$ and $\left\langle\mathbf{I S}_{6}, \uparrow a\right\rangle$ are reduced, while $\left\langle\mathbf{I S}_{6},\{\hat{1}\}\right\rangle$ and $\left\langle\mathbf{I S}_{6}, \uparrow 0\right\rangle$ are not. The reduction of $\left\langle\mathbf{I S}_{6},\{\hat{1}\}\right\rangle$ is isomorphic to $\left\langle\mathbf{I S}_{3},\{\hat{1}\}\right\rangle$, and is therefore isomorphic to a submatrix of $\left\langle\mathbf{I S}_{6}, \uparrow b\right\rangle$. The reduction of $\left\langle\mathbf{I S}_{6}, \uparrow 0\right\rangle$ is isomorphic to $\left\langle\mathbf{I S}_{3},\{0, \hat{1}\}\right\rangle$, which in turn is isomorphic to a submatrix of $\left\langle\mathbf{I S}_{6}, \uparrow a\right\rangle$. Thus $\log \left\{\left\langle\mathbf{I S}_{6}, \uparrow a\right\rangle,\left\langle\mathbf{I S}_{6}, \uparrow 1\right\rangle,\left\langle\mathbf{I S}_{6},\{\hat{1}\}\right\rangle,\left\langle\mathbf{I S}_{6}, \uparrow 0\right\rangle\right\}=\log \left\{\left\langle\mathbf{I S}_{6}, \uparrow a\right\rangle,\left\langle\mathbf{I S}_{6}, \uparrow 1\right\rangle\right\} . \quad$ To conclude the proof, it suffices to show that $\log \left\langle\mathbf{I S}_{6}, \uparrow a\right\rangle \subseteq \log \left\langle\mathbf{I S}_{6}, \uparrow 1\right\rangle$. To see this, notice that $\uparrow 1=\uparrow a \cap \uparrow b$. This easily entails that $\log \left\{\left\langle\mathbf{I S}_{6}, \uparrow a\right\rangle,\left\langle\mathbf{I S}_{6}, \uparrow b\right\rangle\right\} \subseteq \log \left\langle\mathbf{I S}_{6}, \uparrow 1\right\rangle$, and we have seen that $\log \left\{\left\langle\mathbf{I S}_{6}, \uparrow a\right\rangle,\left\langle\mathbf{I S}_{6}, \uparrow b\right\rangle\right\}=\log \left\langle\mathbf{I S}_{6}, \uparrow a\right\rangle$.

In our study, it will be useful to be able to work with reduced matrix models of IS-logics. Proposition 4.2 below suggests that these cannot be characterized by simply applying the Blok-Pigozzi algebraization process, but Proposition 4.4 provides sufficient information for our purposes. (For the definitions of selfextensional, protoalgebraic and algebraizable logic, we refer the reader to [15, respectively, Def. 5.25, 6.1 and 3.11).

Proposition 4.2. $\mathcal{I S} \leq$ is selfextensional and non-protoalgebraic (hence, non-algebraizable).

Proof. Selfextensionality simply follows from the observation that two formulas $\varphi, \psi$ are inter-derivable in $\mathcal{I} \mathcal{S}_{\leq}$if and only if the identity $\varphi \approx \psi$ is valid in the variety of IS-algebras. To show that our logic is not protoalgebraic, we verify that the Leibniz operator $\Omega$ is not monotone on matrix models [15, Thm. 6.13]. To see this, observe that the algebra $\mathbf{I S}_{6}$ has (exactly) one non-trivial congruence $\theta$, which identifies the elements $\{0, a, b, 1\}$. It is then 
easy to check that $\boldsymbol{\Omega}_{\mathbf{I S}_{6}}(\{\hat{1}\})=\theta$. On the other hand, the matrix $\left\langle\mathbf{I S}_{6}, \uparrow 1\right\rangle$ is reduced. Hence, the Leibniz operator is not monotone on the matrix models based on $\mathbf{I S}_{6}$.

Remark 4.3. Proposition 4.2 can in fact be slightly strengthened. If we consider the matrices $\left\langle\mathbf{I S}_{4},\{\hat{1}\}\right\rangle$ and $\left\langle\mathbf{I S}_{4}, \uparrow 1\right\rangle$, where $\mathbf{I S}_{4}$ is the four-element subalgebra of $\mathbf{I S}_{6}$ with universe $\{\hat{0}, 0,1, \hat{1}\}$, we can observe that $\left\langle\mathbf{I S}_{4}, \uparrow 1\right\rangle$ is reduced while $\left\langle\mathbf{I S}_{4},\{\hat{1}\}\right\rangle$ is not. Hence the logic determined by these two submatrices (which is obviously stronger than $\mathcal{I S}_{\leq}$) is also non-protoalgebraic. This, in turn, entails that $\mathcal{I} \mathcal{S}_{\leq}$cannot be protoalgebraic.

The following result is an instance of a general result on order-preserving logics (see e.g. [1, Thm. 2.13.iii]).

Proposition 4.4. $\operatorname{Alg}\left(\mathcal{I} \mathcal{S}_{\leq}\right)=$IS.

The next Proposition we characterizes the logic determined by the class of matrices $\{\langle\mathbf{A},\{1\}\rangle: \mathbf{A} \in \mathbb{I S}\}$. The latter (denoted by $\vdash_{\text {IS }}^{1}$ ) is known in the algebraic logic literature as the 1-assertional logic of the class IS.

Proposition 4.5. $\vdash_{\mathbb{V}\left(\mathbf{I S}_{3}\right)}^{1}=\vdash^{1} \mathrm{IS}=\log \left\langle\mathbf{I S}_{3},\{1\}\right\rangle$.

Proof. Obviously $\vdash_{\mathbb{V}\left(\mathbf{I S}_{3}\right)}^{1} \subseteq \vdash_{\text {IS }}^{1} \subseteq \log \left\langle\mathbf{I S}_{3},\{1\}\right\rangle$, so it suffices to verify the inequality $\log \left\langle\mathbf{I} \mathbf{S}_{3},\{1\}\right\rangle \subseteq \vdash_{\mathbb{V}\left(\mathbf{I S}_{3}\right)}^{1}$. Assume $\Gamma \vdash_{\log \left\langle\mathbf{I} \mathbf{S}_{3},\{1\}\right\rangle} \varphi$. Observe that, since $\log \left\langle\mathbf{I} \mathbf{S}_{3},\{1\}\right\rangle$ is finitary, we can assume $\Gamma$ to be finite. Then $\gamma \vdash_{\log \left\langle\mathbf{I} \mathbf{S}_{3},\{1\}\right\rangle} \varphi$ for $\gamma:=\bigwedge \Gamma$. The latter is equivalent to $\gamma \vdash_{\log \left\langle\mathbf{I S}_{6},\{1\}\right\rangle} \varphi$, because, as observed earlier, $\left\langle\mathbf{A}_{6},\{1\}\right\rangle^{*}=\left\langle\mathbf{I} \mathbf{S}_{3},\{1\}\right\rangle$. In turn, $\gamma \vdash_{\log \left\langle\mathbf{A}_{6},\{1\}\right\rangle} \varphi$ entails that $\mathbf{I S}_{6}$ satisfies the quasi-identity $\gamma \approx \top \Rightarrow \varphi \approx \top$. By Proposition 3.3, this entails that $\gamma \approx \top \Rightarrow \varphi \approx \top$ is satisfied by every $\mathbf{A} \in$ IS. Hence, $\gamma \vdash \varphi$ holds in every matrix in the class $\{\langle\mathbf{A},\{1\}\rangle: \mathbf{A} \in \mathbf{I S}\}$ and, a fortiori, in every matrix in the class $\left\{\langle\mathbf{A},\{1\}\rangle: \mathbf{A} \in \mathbb{V}\left(\mathbf{I S}_{3}\right)\right\}$. This means that $\gamma \vdash_{\mathbf{I S}_{3}}^{1} \varphi$ or, equivalently, $\Gamma \vdash_{\mathbf{I S}_{3}}^{1} \varphi$.

Recalling that the algebra $\mathbf{I S}_{3}$ is isomorphic to to the three-element Eukasiewicz(-Moisil) algebra, Proposition 4.5 tells us that $\vdash_{\text {IS }}^{1}$ is (term equivalent) to three-valued Eukasiewicz logic. This logic is axiomatized, relatively to $\mathcal{I S}_{\leq}$, in Theorem 5.14 (i).

We now return to the construction introduced at the end of Section 3 and illustrate its remarkable logical consequences. Let $\mathbb{M}=\langle\mathbf{A}, D\rangle$ be a matrix, with $\mathbf{A}$ an algebra in the language of De Morgan algebras. Then $\mathbf{A}^{\nabla}$ is in the language of IS. Denoting by $\widehat{\mathbf{A}}$ the $\nabla$-free reduct of $\mathbf{A}^{\nabla}$, let $\widehat{\mathbb{M}}:=\langle\widehat{\mathbf{A}}, D \cup\{\hat{1}\}\rangle$.

Lemma 4.6. Let $\mathbb{M}$ be a reduced model of $\mathcal{B}$. Then $\mathbb{M} \cong(\widehat{\mathbb{M}})^{*}$.

Proof. Recall from [14, Thm. 3.14] that all reduced models of $\mathcal{B}$ are matrices $\mathbb{M}=\langle\mathbf{A}, F\rangle$, with $\mathbf{A}$ a De Morgan algebra and $F$ a lattice filter. To establish the claim, it suffices to show that $\boldsymbol{\Omega}_{\widehat{\mathbf{A}}}(F \cup\{\hat{1}\})=I d_{\widehat{A}} \cup\{\langle 0, \hat{0}\rangle,\langle\hat{0}, 0\rangle,\langle 1, \hat{1}\rangle,\langle\hat{1}, 1\rangle\}$. Let $a, b \in \widehat{A}$. According to [14, Prop. 3.13], we have $\langle a, b\rangle \in \boldsymbol{\Omega}_{\widehat{\mathbf{A}}}(F \cup\{\hat{1}\})$ if and only if, for all $c \in \widehat{A}$, the following hold: $(a \vee c \in F \cup\{\hat{1}\}$ iff $b \vee c \in F \cup\{\hat{1}\})$ and $(\sim a \vee c \in F \cup\{\hat{1}\}$ iff $\sim b \vee c \in F \cup\{\hat{1}\})$. Let us show that $\langle 1, \hat{1}\rangle \in \boldsymbol{\Omega}_{\widehat{\mathbf{A}}}(F \cup\{\hat{1}\})$. Observe that $1 \vee c, \hat{1} \vee c \in F \cup\{\hat{1}\}$ for all $c \in \widehat{A}$. The first condition is thus obviously satisfied. As to the second, assume $\sim 1 \vee c=0 \vee c \in F \cup\{\hat{1}\}$ for some $c \in \widehat{A}$. Then $c \notin\{0, \hat{0}\}$, because $0 \vee \hat{0}=0 \vee 0=0 \notin F \cup\{\hat{1}\}$. This entails $0<c$, so $0 \vee c=c \in F \cup\{\hat{1}\}$. Hence, $\sim \hat{1} \vee c \in F \cup\{\hat{1}\}$. Conversely, if $\sim \hat{1} \vee c=\hat{0} \vee c \in F \cup\{\hat{1}\}$, then we immediately have $\sim 1 \vee c=0 \vee c \in F \cup\{\hat{1}\}$ because $\hat{0} \leq 0$. Hence, $\langle 1, \hat{1}\rangle \in \boldsymbol{\Omega}_{\widehat{\mathbf{A}}}(F \cup\{\hat{1}\})$. By the congruence properties, this entails $\langle\sim 1, \sim \hat{1}\rangle=\langle 0, \hat{0}\rangle \in \boldsymbol{\Omega}_{\widehat{\mathbf{A}}}(F \cup\{\hat{1}\})$, thus also $\langle\hat{0}, 0\rangle,\langle\hat{1}, 1\rangle \in \boldsymbol{\Omega}_{\widehat{\mathbf{A}}}(F \cup\{\hat{1}\})$. Now let $\langle a, b\rangle \in \boldsymbol{\Omega}_{\widehat{\mathbf{A}}}(F \cup\{\hat{1}\})$ be such that $a, b \notin\{\hat{0}, \hat{1}\}$ and 
$a \neq b$. The latter assumption entails that $\langle a, b\rangle \notin \Omega_{\mathbf{A}}(F)$, because $\mathbb{M}$ was reduced. Then, by [14, Prop. 3.13], there is $c \in A$ such that either $(a \vee c \in F$ and $b \vee c \notin F)$ or $(\sim a \vee c \notin F$ and $\sim b \vee c \in F)$. In the former case, we have $a \vee c \in F \cup\{\hat{1}\}$ and $b \vee c \notin F \cup\{\hat{1}\}$, because $b \vee c \neq \hat{1}$ for all $b, c \in A$. Hence, we should have $\langle a, b\rangle \in \boldsymbol{\Omega}_{\widehat{\mathrm{A}}}(F \cup\{\hat{1}\})$, contradicting our assumptions. A similar reasoning shows that the latter case also leads to a contradiction.

We note that Lemma 4.6 could be proved in a more general form, that we shall however not need for our present purposes. Indeed, given an arbitrary (not necessarily reduced) model $\mathbb{M}$ of $\mathcal{B}$, one can show that $\mathbb{M}^{*} \cong(\widehat{\mathbb{M}})^{*}$.

Corollary 4.7. Given matrices $\mathbb{M}$ and $\widehat{\mathbb{M}}$ as per Lemma 4.6, we have $\log \mathbb{M}=\log \widehat{\mathbb{M}}$.

As before, $\mathbb{M}=\langle\mathbf{A}, F\rangle$ is a matrix such that $\mathbf{A}$ is a De Morgan algebra and $F \subseteq A$ a lattice filter on $\mathbf{A}$. Consider the IS-algebra $\mathbf{A}^{\nabla}$ defined according to Proposition 3.4 and let $\mathbb{M}^{\nabla}=\left\langle\mathbf{A}^{\nabla}, F \cup\{\hat{1}\}\right\rangle$.

Corollary 4.8. Let $\mathbb{M}=\langle\mathbf{A}, F\rangle$ be a reduced matrix, with $\mathbf{A}$ a De Morgan algebra and $F \subseteq A$ a lattice filter on $\mathbf{A}$. Then $\log \mathbb{M}^{\nabla}$ is a conservative expansion of $\log \mathbb{M}$.

Proof. Using Corollary 4.7, it suffices to observe that the $\nabla$-free fragment of $\log \mathbb{M}^{\nabla}$ is $\log \widehat{\mathbb{M}}$.

Corollary 4.9. $\mathcal{I S}_{\leq}$is a conservative expansion of the Belnap-Dunn logic $\mathcal{B}$.

Proof. Recall that $\mathcal{I S}_{\leq}=\log \left\langle\mathbf{I S}_{6}, \uparrow a\right\rangle$ (Proposition 4.1), and observe that the matrix $\left\langle\mathbf{I S}_{6}, \uparrow a\right\rangle$ can be obtained as $\mathbb{M}^{\nabla}$ from the four-element matrix $\mathbb{M}=\left\langle\mathbf{D M}_{4}, \uparrow a\right\rangle$ that defines $\mathcal{B}$. Then the result follows from Corollary 4.8 .

Recall that all reduced matrices for the Belnap-Dunn logic (hence, also all reduced matrices for super-Belnap logics) are of the form $\langle\mathbf{A}, F\rangle$, with $\mathbf{A}$ a De Morgan algebra and $F$ a lattice filter [14, Thm. 3.14]. Thus, Lemma 4.6 and Corollaries 4.7 and 4.8 apply, and the latter gives us the following result.

Corollary 4.10. Let $\mathbb{M}_{1}=\left\langle\mathbf{A}_{1}, F_{1}\right\rangle$ and $\mathbb{M}_{2}=\left\langle\mathbf{A}_{2}, F_{2}\right\rangle$ be reduced matrices for the BelnapDunn logic. If $\log \mathbb{M}_{1}^{\nabla}=\log \mathbb{M}_{2}^{\nabla}$, then $\log \mathbb{M}_{1}=\log \mathbb{M}_{2}$.

Given a super-Belnap logic $\vdash$, let $\vdash^{\nabla}=\log \left\{\mathbb{M}^{\nabla}: \mathbb{M} \in \operatorname{Matr}^{*}(\vdash)\right\}$, where each $\mathbb{M}^{\nabla}=$ $\left\langle\mathbf{A}^{\nabla}, F \cup\{\hat{1}\}\right\rangle$ is defined as before. Since $F \cup\{\hat{1}\}$ is a lattice filter of $\mathbf{A}^{\nabla}$, every $\mathbb{M}^{\nabla}$ is a model of $\mathcal{I S}_{\leq}$. Therefore, each logic $\vdash^{\nabla}$ is an extension of $\mathcal{I} \mathcal{S}_{\leq}$.

Lemma 4.11. Let $\vdash$ be a super-Belnap logic. Then $\vdash^{\nabla}$ is a conservative expansion of $\vdash$.

Proof. Suppose, in view of a contradiction, that there exist formulas $\Gamma, \varphi$ in the $\nabla$-free language such that $\Gamma \vdash \varphi$ holds in $\vdash^{\nabla}$ but does not hold in $\vdash$. Then there is $\mathbb{M} \in \operatorname{Matr}^{*}(\vdash)$ such that $\Gamma \nvdash_{\mathbb{M}} \varphi$. Then, by Corollary [4.8, we have that $\Gamma \nvdash_{\mathbb{M} \nabla} \varphi$. By definition, $\vdash^{\nabla} \subseteq$ $\log \mathbb{M}^{\nabla}$. Hence, $\Gamma \nvdash^{\nabla} \varphi$.

Corollary 4.12. Let $\vdash_{1}, \vdash_{2}$ be super-Belnap logics. Then $\vdash_{1} \subseteq \vdash_{2}$ if and only if $\vdash_{1}^{\nabla} \subseteq \vdash_{2}^{\nabla}$.

Proof. Assuming $\vdash_{1} \subseteq \vdash_{2}$, we have $\operatorname{Matr}^{*}\left(\vdash_{2}\right) \subseteq \operatorname{Matr}^{*}\left(\vdash_{1}\right)$. Hence, $\left\{\mathbb{M}^{\nabla}: \mathbb{M} \in \operatorname{Matr}^{*}\left(\vdash_{2}\right.\right.$ )$\} \subseteq\left\{\mathbb{M}^{\nabla}: \mathbb{M} \in \operatorname{Matr}^{*}\left(\vdash_{1}\right)\right\}$, which entails $\vdash_{1}^{\nabla} \subseteq \vdash_{2}^{\nabla}$. Conversely, let $\vdash_{1}^{\nabla} \subseteq \vdash_{2}^{\nabla}$, and let $\Gamma, \varphi$ be formulas (in the language of $\mathcal{B}$ ) such that $\Gamma \vdash_{1} \varphi$. The latter assumption gives us that $\Gamma \vdash_{1}^{\nabla} \varphi$ and, therefore, also $\Gamma \vdash_{2}^{\nabla} \varphi$. Then, by Lemma 4.11, we conclude $\Gamma \vdash_{2} \varphi$. 
Corollary 4.13. The map given by $\vdash \vdash^{\nabla}$ is an embedding of the lattice of super-Belnap logics into the lattice of extensions of $\mathcal{I S}_{\leq}$.

Corollary 4.14. The lattice of extensions of $\mathcal{I} \mathcal{S}_{\leq}$has (at least) the cardinality of the continuum.

Proof. By Corollary 4.13 and the observation that the lattice of super-Belnap logics contains continuum many logics [1, Thm. 4.13].

In fact, in the light of the results of Section 5, we shall be able to prove that there are at least continuum many finitary extensions of $\mathcal{I} \mathcal{S}_{\leq}$.

\section{Axiomatizing IS-LOGiCS}

In 6. 7, the logic $\mathcal{I} \mathcal{S}_{\leq}$is axiomatized by means of a Gentzen calculus. In this Section we tackle the problem of axiomatizing $\mathcal{I} \mathcal{S}_{\leq}$and its extensions by means of Hilbert calculi. From a technical point of view, we shall take profit from the theory of multiple-conclusion calculi, a generalization of traditional Hilbert-style calculi in which the inference rules can have more than one conclusion (with a disjunctive reading). In these calculi proofs are typically ramified instead of sequential. Multiple-conclusion calculi can be used to study single conclusion logics, but also correspond to a generalized notion of logic due to D. Scott and developed by D.J. Shoesmith and T.J. Smiley. We recall some of the basic definitions and results below; for further details see [22, 18.

A multiple-conclusion consequence relation (logic) is a relation $\triangleright \subseteq \wp F m \times \wp F m$ satisfying the following conditions. For every $\Gamma, \Gamma^{\prime}, \Delta, \Delta^{\prime}, \Lambda, T, F \subseteq F m$,

(i) $\Gamma \triangleright \Delta$ whenever $\Gamma \cap \Delta \neq \emptyset$ (overlap),

(ii) $\Gamma, \Gamma^{\prime} \triangleright \Delta, \Delta^{\prime}$ whenever $\Gamma \triangleright \Delta$ (dilution),

(iii) $\Gamma \triangleright \Delta$ whenever $\Gamma, T \triangleright \Delta, F$ for every partition $\langle T, F\rangle$ of $\Lambda$ (cut for sets),

(iv) $\Gamma^{\sigma} \triangleright \Delta^{\sigma}$ for every substitution $\sigma$ whenever $\Gamma \triangleright \Delta$ (substitution invariance).

Given a set of multiple-conclusion rules $\mathrm{R} \subseteq \wp F m \times \wp F m$, we denote by $\triangleright_{\mathrm{R}}$ the smallest multiple-conclusion consequence relation containing $\mathrm{R}$ (hence, $\mathrm{R}$ axiomatizes $\triangleright_{\mathrm{R}}$ ). From a proof-theoretic perspective, we have $\Gamma \triangleright_{\mathrm{R}} \Delta$ whenever there is a labelled tree-proof whose root is labelled by $\Gamma$ and the leaf of every non-discontinued branch is labelled with a formula in $\Delta$. Every class of matrices $\mathcal{M}$ determines a multiple-conclusion logic defined as follows: we let $\Gamma \triangleright_{\mathcal{M}} \Delta$ whenever, for every valuation $v \in \operatorname{Val}(\mathbb{M})$ over a matrix $\mathbb{M}=\langle\mathbf{A}, D\rangle \in \mathcal{M}$, we have that $v(\Gamma) \subseteq D$ implies $v(\Delta) \cap D \neq \emptyset$.

Multiple-conclusion logics smoothly generalize Tarskian logics and their proof-theoretic and semantical definitions. Indeed, for every multiple-conclusion logic $\triangleright$, we have that $\vdash_{\triangleright}=\triangleright \cap(\wp L \times L)$ is a Tarskian consequence relation [15, Def. 1.5]. We call $\vdash_{\triangleright}$ the single-conclusion companion of $\triangleright$ and, given a set of multiple-conclusion rules $\mathrm{R}$, we shall write $\vdash_{R}$ instead of $\vdash_{\triangleright_{R}}$. The following remark contains a few useful facts that can be easily deduced from Sections 5.2 and 17.3 of [22].

Remark 5.1. The sign of a multiple-conclusion relation $\triangleright$ is negative if $F m \triangleright \emptyset$. and is positive otherwise. We denote by $\simeq$ the equivalence relation that identifies two logics $\triangleright_{1}$ and $\triangleright_{2}$ that may differ only in the sign, that is, we let $\triangleright_{1} \simeq \triangleright_{2}$ whenever $\triangleright_{1} \cup$ $\{(F m, \emptyset)\}=\triangleright_{2} \cup\{(F m, \emptyset)\}$. Let $\triangleright_{1} \simeq \triangleright_{2}$. Then $\vdash_{\triangleright_{1}}=\vdash_{\triangleright_{2}}$ and also, if $\triangleright_{1} \subseteq \triangleright \subseteq \triangleright_{2}$, then $\triangleright_{1} \simeq \triangleright_{2} \simeq \triangleright$. Let $\mathbb{P}(\mathcal{M})$ be the closure under products of the class $\mathcal{M}$ (products among matrices are defined as usual for first-order structures; see e.g. [15, p. 225-6]). The following observations are well known: 
(i) $\vdash_{\triangleright_{\mathcal{M}}}=\log \mathcal{M}=\log \mathbb{P}(\mathcal{M})$.

(ii) If $\log \mathcal{M}=\vdash_{\mathrm{R}}$ for $\mathrm{R} \subseteq \wp(L) \times L$, then $\triangleright_{\mathrm{R}} \simeq \triangleright_{\mathbb{P}(\mathcal{M})}$. Therefore, if $\log \mathcal{M}_{1}=\log \mathcal{M}_{2}$ then $\triangleright_{\mathbb{P}\left(\mathcal{M}_{1}\right)} \simeq \triangleright_{\mathbb{P}\left(\mathcal{M}_{2}\right)}$.

Under certain conditions, a (finite) single-conclusion axiomatization can be obtained algorithmically from a (finite) multiple-conclusion axiomatization. The following result covers the case of some of the logics that interest us here.

Given a finite set $\Phi=\left\{\varphi_{1}, \ldots, \varphi_{n}\right\} \subseteq F m$ and $\psi \in F m$, let $\bigvee \Phi:=\left(\left(\varphi_{1} \vee \varphi_{2}\right) \vee \ldots\right) \vee \varphi_{n}$, and let $\Phi \vee \psi=\{\varphi \vee \psi: \varphi \in \Phi\}$.

Theorem 5.2. 22, Thm. 5.37] Let $\mathrm{R}$ be a set of multiple-conclusion rules. Suppose $\vdash_{D_{\mathrm{R}}}$ satisfies, for all $\Gamma \cup\{\varphi, \psi, \xi\} \subseteq F m$, the following property: $\Gamma, \varphi \vee \psi \vdash_{\triangleright_{\mathrm{R}}} \xi$ if and only if $\Gamma, \varphi \vdash_{\triangleright_{\mathrm{R}}} \xi$ and $\Gamma, \psi \vdash_{\triangleright_{\mathrm{R}}} \xi$. Then $\vdash_{\triangleright_{\mathrm{R}}}$ is axiomatized by the set $\mathrm{R}^{\vee}$ consisting of the following rules:

(i) $r^{\vee}=\frac{\bar{\varphi}}{\text { for each } \mathrm{r}=\bar{\varphi}} \in \mathrm{R}$,

(ii) $\mathrm{r}^{\vee}=\frac{\Gamma \vee p_{0}}{(\nabla \Delta) \vee p_{0}}$ for each $\mathrm{r}=\frac{\Gamma}{\Delta} \in \mathrm{R}$,

(iii) $\frac{p}{p \vee q}, \frac{p \vee q}{q \vee p}, \frac{p \vee p}{p}$ and $\frac{p \vee(q \vee r)}{(p \vee q) \vee r}$

where $p_{0}$ is a variable not occurring in $\mathrm{R}$.

We now proceed to explain how the results of Section 4 together with the general considerations on multiple-conclusion logics introduced above are going to be help us deal with extensions of $\mathcal{I} \mathcal{S}_{\leq}$.

5.1. Adding $\nabla$ to the Belnap-Dunn logic. Let $\Sigma=\{\wedge, \vee, \sim, \perp, \top\}$ be the language of $\mathcal{B}$, and let $\Sigma^{\nabla}$ be the expansion of $\Sigma$ with the unary connective $\nabla$ (i.e. the language of $\left.\mathcal{I} \mathcal{S}_{\leq}\right)$. Given a $\Sigma$-matrix $\mathbb{M}=\langle\mathbf{A}, D\rangle$, let $\mathbb{M}^{\nabla}=\left\langle\mathbf{A}^{\nabla}, D \cup\{\hat{1}\}\right\rangle$ be the $\Sigma^{\nabla}$-matrix with underlying algebra $\mathbf{A}^{\nabla}$ defined as in Section 3 (cf. Propositiion 3.4). Let us denote by $\widehat{\mathbb{M}}$ the $\Sigma$-fragment of $\mathbb{M}^{\nabla}$. Observe that, if $\mathbb{M}=\langle\mathbf{A}, D\rangle$ with $\mathbf{A}$ a De Morgan algebra, then $\widehat{\mathbb{M}}$ is precisely the matrix considered in Corollary 4.7. Given a class of $\Sigma$-matrices $\mathcal{M}$, we let $\mathcal{M}^{\nabla}:=\left\{\mathbb{M}^{\nabla}: \mathbb{M} \in \mathcal{M}\right\}$ and $\widehat{\mathcal{M}}:=\{\widehat{\mathbb{M}}: \mathbb{M} \in \mathcal{M}\}$.

The following Theorem contains a generic recipe for axiomatizing the multiple-conclusion logic determined by the class $\mathcal{M}^{\nabla}$, assuming we have a set of rule $\mathrm{R}$ that axiomatizes the multiple-conclusion logic determined by $\widehat{\mathcal{M}}$.

Theorem 5.3. Let $\mathcal{M}$ be a class of $\Sigma$-matrices. If $\triangleright_{\widehat{\mathcal{M}}} \simeq \triangleright_{\mathrm{R}}$, then $\triangleright_{\mathcal{M} \nabla}=\triangleright_{\mathrm{R} \cup \mathrm{R}_{\nabla}}$, where $\mathrm{R}_{\nabla}$ consists of the following rules:

$$
\begin{array}{cccc}
\frac{\nabla p}{\nabla p, \sim \nabla p} \mathrm{r}_{1} & \frac{\nabla p}{\sim \nabla \sim \nabla p} \mathrm{r}_{2} & \frac{\nabla \nabla p}{\nabla p} \mathrm{r}_{3} & \underline{\nabla p, \sim \nabla p} \mathrm{r}_{4} \\
\frac{\sim \nabla p}{\nabla \sim p} \mathrm{r}_{5} & \frac{\nabla \sim \sim p}{\nabla p} \mathrm{r}_{6} & \frac{\nabla p}{\nabla \sim \sim p} \mathrm{r}_{7} \\
\frac{\nabla(p \wedge q)}{\nabla p} \mathrm{r}_{8} & \frac{\nabla(p \wedge q)}{\nabla q} \mathrm{r}_{9} & \frac{\nabla \sim(p \wedge q)}{\nabla \sim p, \nabla \sim q} \mathrm{r}_{10} \\
\frac{\nabla \sim p}{\nabla \sim(p \wedge q)} \mathrm{r}_{11} & \frac{\nabla \sim q}{\nabla \sim(p \wedge q)} \mathrm{r}_{12} & \frac{\nabla p, \nabla q}{\nabla(p \wedge q)} \mathrm{r}_{13}
\end{array}
$$




$$
\begin{gathered}
\frac{\nabla \sim(p \vee q)}{\nabla \sim p} \mathrm{r}_{14} \quad \frac{\nabla \sim(p \vee q)}{\nabla \sim q} \mathrm{r}_{15} \quad \frac{\nabla(p \vee q)}{\nabla p, \nabla q} \mathrm{r}_{16} \\
\frac{\nabla p}{\nabla(p \vee q)} \mathrm{r}_{17} \quad \frac{\nabla q}{\nabla(p \vee q)} \mathrm{r}_{18} \quad \frac{\nabla \sim p, \nabla \sim q}{\nabla \sim(p \vee q)} \mathrm{r}_{19} \\
\frac{\sim \nabla \perp}{\sim \nabla 2} \mathrm{r}_{20} \quad \frac{\overline{\sim \nabla \sim \top}}{\mathrm{r}_{21}}
\end{gathered}
$$

Proof. Checking the soundness of the new rules is routine. We give only a couple of examples. Let $v$ be a valuation over a matrix $\mathbb{M}^{\nabla}$. The rule $r_{1}$ is sound in $\mathbb{M}^{\nabla}$, for either $v(\nabla \varphi)=\hat{1}$ (if $v(\varphi) \neq \hat{0}$ ) or $v(\sim \nabla \varphi)=\hat{1}$ (if $v(\varphi)=\hat{0}$ ). Regarding rule $\mathrm{r}_{2}$, we have that, if $v(\nabla \varphi)=\hat{1}$ then $v(\sim \nabla \varphi)=v(\nabla \sim \nabla \varphi)=\hat{0}$, so $v(\sim \nabla \sim \nabla \varphi)=\hat{1}$.

For completeness, assume $\Gamma \phi_{\mathrm{R}_{\nabla}} \Delta$. Then, by cut for sets, there is a partition $\langle T, F\rangle$ of $L_{\Sigma \nabla}$ such that $\Gamma \subseteq T$ and $\Delta \subseteq F$ and $T \ngtr_{\mathrm{R}_{\nabla}} F$. Note that (by $\mathrm{r}_{1}$ and $\mathrm{r}_{4}$ ) for each $\varphi$, we have either $\nabla \varphi \in T$ or $\sim \nabla \varphi \in T$, but never both. In particular, $F$ is never empty. Also, by $\mathrm{r}_{5}$ we must have either $\nabla \varphi \in T$ or $\nabla \sim \varphi \in T$. Hence, each $\varphi$ must be exactly in one of three cases: (i) $\nabla \varphi, \nabla \sim \varphi \in T$, (ii) $\sim \nabla \sim \varphi \in T$, or (iii) $\sim \nabla \varphi \in T$.

Since $\mathrm{R} \subseteq \mathrm{R} \cup \mathrm{R}_{\nabla}$, we also have $T \ngtr_{\mathrm{R}} \quad F$. From the fact that $\triangleright_{\mathrm{R}} \simeq \triangleright_{\widehat{\mathbb{M}}}$ and $F \neq \emptyset$ we know that $T \not_{\widehat{\mathbb{M}}} F$. We can therefore pick $v \in \operatorname{Hom}_{\Sigma}\left(L_{\Sigma^{\nabla}}, \widehat{\mathbb{M}}\right)$, for some $\mathbb{M} \in \mathcal{M}$ such that $v(T) \subseteq D$ and $v(F) \cap D=\emptyset$. Consider $v^{\prime}: L_{\Sigma^{\nabla}} \rightarrow \mathbb{M}^{\nabla}$ defined by:

$$
v^{\prime}(\varphi):= \begin{cases}\hat{1} & \text { if } \sim \nabla \sim \varphi \in T \\ \hat{0} & \text { if } \sim \nabla \varphi \in T \\ v\left(\varphi_{i}\right) & \text { if } \varphi=\varphi_{1} \wedge \varphi_{2} \text { and } \sim \nabla \sim \varphi_{3-i} \in T \\ \left.v \varphi_{i}\right) & \text { if } \varphi=\varphi_{1} \vee \varphi_{2} \text { and } \sim \nabla \varphi_{3-i} \in T \\ v(\varphi) & \text { if } \nabla \varphi, \nabla \sim \varphi \in T\end{cases}
$$

We will show that $v^{\prime} \in \operatorname{Val}\left(\mathbb{M}^{\nabla}\right)=\operatorname{Hom}_{\Sigma^{\nabla}}\left(L_{\Sigma^{\nabla}}, \widehat{\mathbb{M}}\right)$.

1. $v^{\prime}(\nabla \varphi)=\nabla v^{\prime}(\varphi)$

From $r_{1}$ we have that either (ii) $\nabla \varphi \in T$ or (iii) $\sim \nabla \varphi \in T$.

If (ii) $\nabla \varphi \in T$, by $\mathrm{r}_{4}$ we have that $\sim \nabla \varphi \notin T$ and so $v^{\prime}(\varphi) \neq \hat{0}$. Further, by $\mathrm{r}_{2}$ we obtain that $\sim \nabla \sim \nabla \varphi \in T$, hence $v^{\prime}(\nabla \varphi)=\hat{1}=\nabla\left(v^{\prime}(\varphi)\right)$.

If instead iii) $\sim \nabla \varphi \in T$ then $v^{\prime}(\varphi)=\hat{0}$ and by $\mathrm{r}_{4}$ we have that $\nabla \varphi \notin T$. Hence, by $\mathrm{r}_{3}, \nabla \nabla \varphi \notin T$, and by $\mathrm{r}_{1}$ we get that $\sim \nabla \nabla \varphi \in T$ and $v^{\prime}(\nabla \varphi)=\hat{0}=\nabla\left(v^{\prime}(\varphi)\right)$.

2. $v^{\prime}(\sim \varphi)=\sim v^{\prime}(\varphi)$

If (i) $\nabla \sim \varphi, \nabla \sim \sim \varphi \in T$ then by $\mathrm{r}_{6}, \nabla \varphi \in T$ (so $v^{\prime}(\varphi)=v(\varphi)$ ) and therefore $v^{\prime}(\sim \varphi)=v(\sim \varphi)=\sim_{\mathbb{M}}(v(\varphi))=\tilde{\sim}\left(v^{\prime}(\varphi)\right)$.

If (ii) $\sim \nabla \sim \sim \varphi \in T$ (so $v^{\prime}(\sim \varphi)=\hat{1}$ ) then by $\mathrm{r}_{1}$ and $\mathrm{r}_{7}$ we have that $\sim \nabla \varphi \in T$ (so $\left.v^{\prime}(\varphi)=\hat{0}\right)$ hence $v^{\prime}(\sim \varphi)=\hat{1}=\tilde{\sim}\left(v^{\prime}(\varphi)\right)$.

If (iii) $\sim \nabla \sim \varphi \in T$ then $v^{\prime}(\sim \varphi)=\hat{0}$ and $v^{\prime}(\varphi)=\hat{1}$, thus we immediately obtain $v^{\prime}(\sim \varphi)=\sim v^{\prime}(\varphi)$

3. $v^{\prime}(\varphi \wedge \psi)=v^{\prime}(\varphi) \wedge v^{\prime}(\varphi)$

If (i) we have that $\nabla(\varphi \wedge \psi), \nabla \sim(\varphi \wedge \psi) \in T$. From $\nabla(\varphi \wedge \psi) \in T$, by $\mathbf{r}_{8}$ and $\mathbf{r}_{9}$ we obtain that $\nabla \varphi, \nabla \psi \in T\left(v^{\prime}(\varphi) \neq \hat{0} \neq v^{\prime}(\psi)\right)$. Also, from $\nabla \sim(\varphi \wedge \psi) \in T$, by

$\mathrm{r}_{10}$, either $\nabla \sim \varphi$ or $\nabla \sim \psi$ are in $T\left(v^{\prime}(\varphi) \neq \hat{1}\right.$ or $\left.v^{\prime}(\psi) \neq \hat{1}\right)$. Hence, if $\nabla \sim \varphi, \nabla \sim \psi \in$ $T$ then $v^{\prime}(\varphi \wedge \psi)=v(\varphi \wedge \psi)=v(\varphi) \tilde{\wedge} v(\psi)=v^{\prime}(\varphi) \tilde{\wedge} v^{\prime}(\varphi)$. Otherwise, if $\nabla \sim \varphi \notin T$, 
then by $\mathrm{r}_{1}$ we conclude that $\sim \nabla \sim \varphi \in T$ and hence $v^{\prime}(\varphi \wedge \psi)=v^{\prime}(\psi)=\hat{1} \tilde{\wedge} v^{\prime}(\psi)=$ $v^{\prime}(\varphi) \tilde{\wedge} v^{\prime}(\psi)$. The case $\nabla \sim \psi \notin T$ is similar.

If (ii) we have that $\sim \nabla \sim(\varphi \wedge \psi) \in T$ (so $v^{\prime}(\varphi \wedge \psi)=\hat{1}$ ) then by $\mathrm{r}_{4}$ we have that $\nabla \sim(\varphi \wedge \psi) \notin T$. By $\mathrm{r}_{11}$ and $\mathrm{r}_{12}$ we have that $\nabla \sim \varphi, \nabla \sim \psi \notin T$. Hence, by $\mathrm{r}_{1}$, $\sim \nabla \sim \varphi, \sim \nabla \sim \psi \in T\left(\right.$ so $\left.v^{\prime}(\varphi)=v^{\prime}(\psi)=\hat{1}\right)$ and $v^{\prime}(\varphi \wedge \psi)=\hat{1}=v^{\prime}(\varphi) \tilde{\wedge} v^{\prime}(\psi)$.

If (iii) we have that $\sim \nabla(\varphi \wedge \psi) \in T$ (so $v^{\prime}(\varphi \wedge \psi)=\hat{0}$ ) then by $\mathrm{r}_{4}, \nabla(\varphi \wedge \psi) \notin T$. By $\mathrm{r}_{13}$ and $\mathrm{r}_{1}$ we have that either $\sim \nabla \varphi \in T$ or $\sim \nabla \psi \in T$ (so either $v^{\prime}(\varphi)=\hat{0}$ or $\left.v^{\prime}(\psi)=\hat{0}\right)$ hence $v^{\prime}(\varphi \wedge \psi)=\hat{0}=v^{\prime}(\varphi) \tilde{\wedge} v^{\prime}(\psi)$.

4. $v^{\prime}(\varphi \vee \psi)=v^{\prime}(\varphi) \vee v^{\prime}(\psi)$

If (i) we have that $\nabla(\varphi \vee \psi), \nabla \sim(\varphi \vee \psi) \in T$. From $\nabla \sim(\varphi \vee \psi) \in T$, by $\mathrm{r}_{14}$ and $\mathrm{r}_{15}$ we obtain that $\nabla \sim \varphi, \nabla \sim \psi \in T\left(v^{\prime}(\varphi) \neq \hat{1} \neq v^{\prime}(\psi)\right)$. From $\nabla(\varphi \vee \psi) \in T$, by $\mathrm{r}_{16}$, either $\nabla \varphi$ or $\nabla \psi$ are in $T\left(v^{\prime}(\varphi) \neq \hat{0}\right.$ or $\left.v^{\prime}(\psi) \neq \hat{0}\right)$. Hence, if $\nabla \varphi, \nabla \psi \in T$ then $v^{\prime}(\varphi \vee \psi)=v(\varphi \vee \psi)=v(\varphi) \tilde{V} v(\psi)=v^{\prime}(\varphi) \tilde{\vee} v^{\prime}(\varphi)$. Otherwise, if $\nabla \varphi \notin T$, then by $\mathrm{r}_{1}$ we conclude that $\sim \nabla \varphi \in T$ and hence $v^{\prime}(\varphi \vee \psi)=v^{\prime}(\psi)=\hat{0} \tilde{\vee} v^{\prime}(\psi)=v^{\prime}(\varphi) \tilde{\wedge} v^{\prime}(\psi)$. The case $\nabla \psi \notin T$ is similar.

If (ii) we have that $\sim \nabla \sim(\varphi \vee \psi) \in T$ (so $v^{\prime}(\varphi \vee \psi)=\hat{1}$ ) then $\sim(\varphi \vee \psi) \notin T$ by $\mathrm{r}_{4}$. From $\mathrm{r}_{19}$ and $\mathrm{r}_{1}$ either $\sim \nabla \sim \varphi \in T$ or $\sim \nabla \sim \psi \in T$. (so either $v^{\prime}(\varphi)=\hat{1}$ or $\left.v^{\prime}(\psi)=\hat{1}\right)$ hence $v^{\prime}(\varphi \vee \psi)=\hat{1}=v^{\prime}(\varphi) \tilde{V} v^{\prime}(\psi)$.

If (iii) we have that $\sim \nabla(\varphi \vee \psi) \in T$ (so $\left.v^{\prime}(\varphi \vee \psi)=\hat{0}\right)$ and so $\nabla(\varphi \vee \psi) \notin T$ by $\mathrm{r}_{4}$. By $\mathrm{r}_{17}, \mathrm{r}_{18}$ we have that $\nabla \varphi, \nabla \psi \notin T$, and by $\mathrm{r}_{1}, \sim \nabla \varphi, \sim \nabla \psi \in T$ (so $v^{\prime}(\varphi)=v^{\prime}(\psi)=\hat{0}$ ). Thus, $v^{\prime}(\varphi \vee \psi)=v^{\prime}(\varphi) \vee v^{\prime}(\psi)=\hat{0} \tilde{\vee} \hat{0}=\hat{0}$.

5. $v^{\prime}(\perp)=\hat{0}$ and $v^{\prime}(\top)=\hat{1}$

Directly from rules $r_{20}$ and $r_{21}$.

Regarding the preceding Proposition, note that, in order to show that $v^{\prime}(\xi)$ is well defined for every $\xi \in F m_{\Sigma^{\nabla}}$ (and that $v^{\prime} \in \operatorname{Val}\left(\mathbb{M}^{\nabla}\right)$ ), we only need to consider rules in $\mathbf{R}_{\nabla}$ instantiated with formulas in $\operatorname{sub}(\xi)$. This observation will be crucial in the proof of Theorem 5.15.

We now proceed to explain how a single-conclusion axiomatization (for a logic extending $\mathcal{I} \mathcal{S}_{\leq}$) can be extracted from the multiple-conclusion rules of Theorem [5.3. We shall need a few technical lemmas. In the next one, $\prod_{i} \mathbb{M}_{i}$ denotes the product of a family of matrices $\left\{\mathbb{M}_{i}: i \in I\right\}$.

Lemma 5.4. Given a class $\left\{\mathbb{M}_{i}: i \in I\right\}$ of $\Sigma$-matrices, we have the following embeddings:

$$
\prod_{i} \mathbb{M}_{i} \hookrightarrow\left(\prod_{i} \mathbb{M}_{i}\right)^{\nabla} \hookrightarrow \prod_{i}\left(\mathbb{M}_{i}^{\nabla}\right) .
$$

Proof. The fist embedding is simply the identity function. The second one is also the identity for the elements in $\prod_{i}\left(\mathbb{M}_{i}\right)$, whereas $\hat{1}$ is sent to $\prod_{i}\{\hat{1}\}$ and $\hat{0}$ to $\prod_{i}\{\hat{0}\}$.

The following result is an immediate consequence of Lemma 5.4

Lemma 5.5. For every class $\mathcal{M}$ of $\Sigma$-matrices, we have:

(i) $\triangleright_{\mathbb{P}(\widehat{\mathcal{M}})} \subseteq \triangleright_{\mathbb{P}(\mathcal{M})} \subseteq \triangleright_{\mathbb{P}(\mathcal{M})}$.

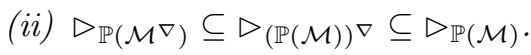

Let $\mathrm{R}$ be a a set of single-conclusion rules. Recall that $\mathrm{R}_{\nabla}$ is a set of multiple-conclusion rules, and we abbreviate $\vdash_{\mathrm{R} \cup \mathrm{R}_{\nabla}}=\vdash_{\triangle_{\mathrm{R} \cup R_{\nabla}}}$. 
Corollary 5.6. Let $\mathcal{M}$ be a class of $\Sigma$-matrices, and let $\mathrm{R}$ be a set of single-conclusion rules. If $\vdash_{\mathrm{R}}=\log \mathcal{M}=\log \widehat{\mathcal{M}}$, then $\vdash_{\mathrm{R} \cup \mathrm{R}_{\nabla}}=\log \mathcal{M}^{\nabla}$.

Proof. From $\log \mathcal{M}=\log \widehat{\mathcal{M}}=\vdash_{R}$ we have $\triangleright_{R} \simeq \triangleright_{\mathbb{P}(\widehat{\mathcal{M}})} \simeq \triangleright_{\mathbb{P}(\mathcal{M})}$ by Remark 5.1 (ii). From Lemma 5.5 (i) and Remark 5.1 we obtain that $\triangleright_{R} \simeq \triangleright_{\mathbb{P}(\mathcal{M})}$. By Theorem 5.3 we conclude that $\triangleright_{(\mathbb{P}(\mathcal{M}))^{\nabla}}=\triangleright_{R^{\prime} \cup R_{\nabla}}$. Finally, from Lemma 5.5 (ii) and Remark 5.1 we have $\log \mathcal{M}^{\nabla}=$ $\log \mathbb{P}(\mathcal{M})^{\nabla}$. Hence $\vdash_{\mathcal{M} \nabla}$ is axiomatized by $\mathrm{R} \cup \mathrm{R}_{\nabla}$.

Joining Theorem 5.2 and Corollary [5.6, we obtain the following recipe for capturing the effect of adding $\nabla$ to single-conclusion axiomatizations.

Corollary 5.7. Let $\mathcal{M}$ be a class of $\Sigma$-matrices and let $\mathrm{R}$ be a set of single-conclusion rules. If $\vdash_{\mathrm{R}}=\log \mathcal{M}=\log \widehat{\mathcal{M}}$, then $\vdash_{\left(R \cup R_{\nabla}\right)^{\vee}}=\log \mathcal{M}^{\nabla}$.

Example 5.8. Let $\mathbb{M}_{4}=\left\langle\mathbf{D M}_{4}, \uparrow a\right\rangle$ be the four-element matrix that defines the BelnapDunn logic $\mathcal{B}$. By Corollary 4.6 we know that $\mathcal{B}=\log \mathbb{M}_{4}=\log \widehat{\mathbb{M}}_{4}$. Hence, from Corollaries 4.7 and 5.7 we can obtain a Hilbert axiomatization for $\mathcal{I S}_{\leq}=\log \mathbb{M}_{4}^{\nabla}=\log \left\langle\mathbf{I S}_{6}, \uparrow a\right\rangle$. Let $R_{\mathcal{B}}$ be the Hilbert-style calculus used in [14 to axiomatize $\log \mathcal{B}$ (expanded with the rules introduced in [1, p. 1065] to account for the constants).

$$
\begin{array}{ccc}
\frac{p \wedge q}{p} & \frac{p \wedge q}{q} & \frac{p}{p \wedge q} \\
\frac{p}{p \vee q} & \frac{p \vee q}{q \vee p} & \frac{p \vee p}{p} \\
\frac{p \vee(q \vee r)}{(p \vee q) \vee r} & \frac{p \vee(q \wedge r)}{(p \vee q) \wedge(p \vee r)} & \frac{(p \vee q) \wedge(p \vee r)}{p \vee(q \wedge r)} \\
\frac{p \vee r}{\neg \neg p \vee r} & \frac{\neg \neg p \vee r}{p \vee r} & \frac{\neg(p \vee q) \vee r}{(\neg p \wedge \neg q) \vee r} \\
\frac{(\neg p \wedge \neg q) \vee r}{\neg(p \vee q) \vee r} & \frac{\neg(p \wedge q) \vee r}{(\neg p \vee \neg q) \vee r} & \frac{(\neg p \vee \neg q) \vee r}{\neg(p \wedge q) \vee r} \\
\bar{\top} & \frac{\perp \perp \vee p}{p} & \frac{\sim \top \vee p}{p}
\end{array}
$$

Then $\mathcal{I} \mathcal{S}_{\leq}$is axiomatized by $\left(R_{\mathcal{B}} \cup R_{\nabla}\right)^{\vee}$, which is the result of adding to $R_{\mathcal{B}}$ the following rules:

$$
\begin{aligned}
& \frac{\nabla p \vee \sim \nabla p}{\nabla} r_{1}^{\vee} \quad \frac{\nabla p \vee r}{\sim \nabla \sim \nabla p \vee r} r_{2}^{\vee} \quad \frac{\nabla \nabla p \vee r}{\nabla p \vee r} r_{3}^{\vee} \quad \frac{\nabla p \vee r, \sim \nabla p \vee r}{r} r_{4}^{\vee} \\
& \frac{\sim \nabla p \vee r}{\nabla \sim p \vee r} r_{5}^{\vee} \quad \frac{\nabla \sim \sim p \vee r}{\nabla p \vee r} r_{6}^{\vee} \quad \frac{\nabla p \vee r}{\nabla \sim \sim p \vee r} r_{7}^{\vee} \\
& \frac{\nabla(p \wedge q) \vee r}{\nabla p \vee r} r_{8}^{\vee} \quad \frac{\nabla(p \wedge q) \vee r}{\nabla q \vee r} r_{9}^{\vee} \quad \frac{\nabla \sim(p \wedge q) \vee r}{\nabla \sim p \vee \nabla \sim q \vee r} r_{10}^{\vee}
\end{aligned}
$$




$$
\begin{gathered}
\frac{\nabla \sim p \vee r}{\nabla \sim(p \wedge q) \vee r} r_{11}^{\vee} \quad \frac{\nabla \sim q \vee r}{\nabla \sim(p \wedge q) \vee r} r_{12}^{\vee} \quad \frac{\nabla p \vee r, \nabla q \vee r}{\nabla(p \wedge q) \vee r} r_{13}^{\vee} \\
\frac{\nabla \sim(p \vee q) \vee r}{\nabla \sim p \vee r} r_{14}^{\vee} \quad \frac{\nabla \sim(p \vee q) \vee r}{\nabla \sim q \vee r} r_{15}^{\vee} \quad \frac{\nabla(p \vee q) \vee r}{\nabla p \vee \nabla q \vee r} r_{16}^{\vee} \\
\frac{\nabla p \vee r}{\nabla(p \vee q) \vee r} r_{17}^{\vee} \quad \frac{\nabla q \vee r}{\nabla(p \vee q) \vee r} r_{18}^{\vee} \frac{\nabla \sim p \vee r, \nabla \sim q \vee r}{\nabla \sim(p \vee q) \vee r} r_{19}^{\vee} \\
\frac{\sim \nabla \perp}{\sim} r_{20}^{\vee} \frac{\sim \nabla \sim \top}{\sim} r_{21}^{\vee}
\end{gathered}
$$

In the next Subsection we are going to apply Corollary 5.7 to axiomatize (relatively to $\mathcal{B}^{\nabla}$ ), some extensions of $\mathcal{I} \mathcal{S}_{\leq}$that are characterized by $\mathbb{M}^{\nabla}$ for some matrix $\mathbb{M}$ that is a model of $\mathcal{B}$.

5.2. Adding $\nabla$ to super-Belnap logics. As observed earlier, $\mathcal{I} \mathcal{S}_{\leq}=\vdash_{\mathcal{M}}$, where $\mathcal{M}$ is the following class of matrices:

$$
\mathcal{M}:=\{\langle\mathbf{A}, F\rangle: \mathbf{A} \in \mathrm{IS}, F \subseteq A \text { is a (non-empty) lattice filter }\} .
$$

Thus, each subclass $\mathcal{M}^{\prime} \subseteq \mathcal{M}$ (it suffices to consider those $\mathcal{M}^{\prime}$ consisting of reduced matrices) defines a logic $\vdash_{\mathcal{M}^{\prime}}$ which is an extension of $\mathcal{I} \mathcal{S}_{\leq}$. We have seen with Corollary 4.14 that there are at least continuum many of these, and Corollary 4.13 suggests that the structure of the lattice of extensions of $\mathcal{I S} \leq$ is quite complex (see [1, 20, for analogous considerations on the lattice of super-Belnap logics). A systematic study of this lattice lies outside the scope of the present paper and even beyond our present grasp on IS-logics; however, in this Subsection we consider a few extensions of $\mathcal{I} \mathcal{S}_{\leq}$that are defined by substructures of $\left\langle\mathbf{I S}_{6}, \uparrow a\right\rangle$, illustrating how our methods can be used to axiomatize them.

The following result, which is an immediate consequence of Corollary [5.7 shows that Example 5.8 smoothly generalizes to all super-Belnap logics.

Proposition 5.9. Let $\mathcal{M}$ be a class of models of $\mathcal{B}$. If $\log \mathcal{M}$ is axiomatized relative to $\mathcal{B}$ by a set of single conclusion rules $\mathrm{R}$, then $\log \mathcal{M}^{\nabla}$ is also axiomatized by $\mathrm{R}$ relative to $\mathcal{B}^{\nabla}$.

Let $\mathcal{M}_{1}$ and $\mathcal{M}_{2}$ be classes of models of $\mathcal{B}$ such that $\log \mathcal{M}_{1}=\log \mathcal{M}_{2}$. Then $\log \mathcal{M}_{1}$ and $\log \mathcal{M}_{2}$ are axiomatized by the same set $\mathrm{R}$ of single-conclusion rules. Hence, $\log \mathcal{M}_{1}^{\nabla}=$ $\log \mathcal{M}_{2}^{\nabla}$ is axiomatized by the set $\mathrm{R}_{\nabla}^{\vee}$ defined above. This entails, in particular, that, if a super-Belnap logic $\vdash$ is finitary (resp. finitely axiomatized), then $\vdash^{\nabla}$ (defined as in Corollary 4.13) is also finitary (resp. finitely axiomatized). Since the lattice of super-Belnap logics contains continuum many finitary logics [20, Cor. 8.17], the above considerations allow us to obtain the following sharpening of Corollary 4.14

Proposition 5.10. There are (at least) continuum many finitary extensions of $\mathcal{I S}_{\leq}$.

The super-Belnap logics considered below are the so-called Exactly True Logic $\mathcal{E} \mathcal{T} \mathcal{L}$ of 19 (which is the 1-assertional logic of the variety of De Morgan algebras), G. Priest's Logic of Paradox $\mathcal{L} \mathcal{P}$, the two logics $\mathcal{K}_{\leq}$and $\mathcal{K}_{1}$ named after $\mathrm{S}$. C. Kleene, and classical $\operatorname{logic} \mathcal{C} \mathcal{L} . \mathcal{K}_{\leq}$is the order-preserving logic of the variety of Kleene algebras, and $\mathcal{K}_{1}$ is the $1-$ assertional logic associated to the same variety (see [1] for further details). Proposition 5.11] 
below shows that each of these logics can be axiomatized, relative to $\mathcal{B}$, by a combination of the following rules:

$$
\begin{gathered}
p \wedge(\sim p \vee q) \vdash q \\
(p \wedge \sim p) \vee q \vdash q \\
(p \wedge \sim p) \vee r \vdash q \vee \sim q \vee r \\
\emptyset \vdash p \vee \sim p
\end{gathered}
$$$$
\left(K_{\leq}\right)
$$

(Regarding the names of the above rules, the $K$ 's are suggestive of Kleene's logics, (DS) stands for Disjunctive Syllogism and (EM) for Excluded Middle.)

Proposition 5.11 (1], Thm. 3.4).

(i) $\mathcal{E} \mathcal{T} \mathcal{L}=\log \left\langle\mathbf{D M}_{4},\{1\}\right\rangle=\mathcal{B}+(\overline{\mathrm{DS}})$.

(ii) $\mathcal{L P}=\log \left\langle\mathbf{K}_{3}, \uparrow a\right\rangle=\mathcal{B}+(\underline{\mathrm{EM}})$.

(iii) $\mathcal{K}_{1}=\log \left\langle\mathbf{K}_{3},\{1\}\right\rangle=\mathcal{B}+K_{1}$.

(iv) $\mathcal{K}_{\leq}=\log \left\{\left\langle\mathbf{K}_{3}, \uparrow a\right\rangle,\left\langle\mathbf{K}_{3},\{1\}\right\rangle\right\}=\mathcal{B}+\left|K_{\leq}\right\rangle$.

(v) $\mathcal{C} \mathcal{L}=\log \left\langle\mathbf{B}_{2},\{1\}\right\rangle=\mathcal{B}+(\overline{D S})+(\mathrm{EM})$.

Theorem 5.12. For logics above $\mathcal{I S}_{\leq}$we have the following relative axiomatizations:

(i) $\log \left\langle\mathbf{I S}_{6}, \uparrow 1\right\rangle=\mathcal{E} \mathcal{T} \mathcal{L}^{\nabla}=\mathcal{I S}_{\leq}+(\overline{\mathrm{DS}})$.

(ii) $\log \left\langle\mathbf{I S}_{5}, \uparrow a\right\rangle=\mathcal{L P}^{\nabla}=\mathcal{I S}_{\leq}+(\overline{E M})$.

(iii) $\log \left\langle\mathbf{I S}_{5}, \uparrow 1\right\rangle=\mathcal{K}_{1}^{\nabla}=\mathcal{I S}_{\leq}+K_{1}$.

(iv) $\log \left\{\left\langle\mathbf{I S}_{5}, \uparrow a\right\rangle,\left\langle\mathbf{I S}_{5}, \uparrow 1\right\rangle\right\}=\mathcal{K}_{\leq}^{\nabla}=\mathcal{I} \mathcal{S}_{\leq}+K_{\leq}$.

(v) $\log \left\langle\mathbf{I S}_{4}, \uparrow 1\right\rangle=\mathcal{C} \mathcal{L}^{\nabla}=\mathcal{I S}_{\leq}+(\overline{\mathrm{DS}})+(\mathrm{EM})$.

Proof. The statement follows directly from Proposition 5.9 and Proposition [5.11, having noticed that, for $x \in\{1, a\}$, we have $\left\langle\mathbf{D M}_{4}, \uparrow x\right\rangle^{\nabla}=\left\langle\mathbf{I S}_{6}, \uparrow x\right\rangle,\left\langle\mathbf{K}_{3}, \uparrow x\right\rangle^{\nabla}=\left\langle\mathbf{I S}_{5}, \uparrow x\right\rangle$ and $\left\langle\mathbf{B}_{2}, \uparrow 1\right\rangle^{\nabla}=\left\langle\mathbf{I S}_{4}, \uparrow 1\right\rangle$.

5.3. Other extensions of $\mathcal{I} \mathcal{S}_{\leq}$. In this Subsection we consider a few examples of extensions of $\mathcal{I} \mathcal{S}_{\leq}$(defined by substructures of the matrix $\left\langle\mathbf{I S}_{6}, \uparrow a\right\rangle$ )

Given a $\Sigma$-matrix $\mathbb{M}=\langle\mathbf{A}, D\rangle$, a set of axioms $\mathrm{Ax} \subseteq F m_{\Sigma}$ and a set of rules $\mathrm{R} \subseteq$ $\wp\left(F m_{\Sigma}\right) \times F m_{\Sigma}$, we write $\operatorname{Val}_{\mathbb{M}}^{A^{A}}$ for the set of valuations on $\mathbb{M}$ such that $v\left(\varphi^{\sigma}\right) \subseteq D$ for every $\varphi \in \mathrm{Ax}$ substitution $\sigma$, and $\operatorname{Val}_{\mathbb{M}}^{\mathbb{R}}$ for the set of valuations on $\mathbb{M}$ such that $v\left(\Gamma^{\sigma}\right) \subseteq D$ implies $\varphi^{\sigma} \in D$ for every $\frac{\Gamma}{\varphi} \in \mathrm{R}$ and substitution $\sigma$.

The following result (whose simple proof we omit) is a corollary of [4, Lemma 2.7] and will be very useful to show relative axiomatization results (this technique is used in [5] to obtain general modular semantics for axiomatic extensions of a given logic). In item (ii), $\mathbb{M}^{\omega}$ is a shorthand for $\prod_{i<\omega} \mathbb{M}$.

Proposition 5.13. Let $\mathbb{M}=\langle\mathbf{A}, D\rangle$ be a $\Sigma$-matrix. Given $\mathrm{Ax} \subseteq F m_{\Sigma}$ and $\mathrm{R} \subseteq \wp\left(F m_{\Sigma}\right) \times$ $F m_{\Sigma}$. We have that:

(i) Val $A_{\mathbb{M}}^{\mathrm{Ax}}$ is a complete semantics for $\log (\mathbb{M})+\mathrm{Ax}$.

(ii) $V a l_{\mathbb{M} \omega}^{R}$ is a complete semantics for $\log (\mathbb{M})+\mathrm{R}$. 
We are now ready to give an axiomatization relative to $\mathcal{I} \mathcal{S}_{\leq}$of $\vdash_{\text {IS }}^{1}$, the 1 -assertional logic of the class IS (i.e. three-valued Eukasiewicz(-Moisil) logic; cf. Proposition 4.5). This is the first item of Theorem 5.14 below. The logic axiomatized by the second item is the order-preserving logic of the variety $\mathbb{V}\left(\mathbf{I S}_{3}\right)$ of three-valued Lukasiewicz(-Moisil) algebras, which is also $\log \left\{\left\langle\mathbf{I S}_{3},\{\hat{1}\}\right\rangle,\left\langle\mathbf{I S}_{3}, \uparrow 0\right\rangle\right\}$.

\section{Theorem 5.14.}

(i) $\log \left\langle\mathbf{I S}_{6},\{\hat{1}\}\right\rangle=\log \left\langle\mathbf{I S}_{5},\{\hat{1}\}\right\rangle=\log \left\langle\mathbf{I S}_{4},\{\hat{1}\}\right\rangle=\log \left\langle\mathbf{I S}_{3},\{\hat{1}\}\right\rangle=\vdash_{\text {IS }}^{1}=\mathcal{I} \mathcal{S}_{\leq}+p \vdash$ $\sim \nabla \sim p$.

(ii) $\log \left\{\left\langle\mathbf{I S}_{3},\{\hat{1}\}\right\rangle,\left\langle\mathbf{I S}_{3}, \uparrow 0\right\rangle\right\}=\vdash_{\mathbb{V}\left(\mathbf{I S}_{3}\right)}^{\leq}=\mathcal{I} \mathcal{S}_{\leq}+\left[K_{\leq}+\sim p \vee r, \nabla p \vee r \vdash p \vee r+\sim \nabla p \vee r \vdash\right.$ $\sim p \vee r$.

(iii) $\log \left(\left\langle\mathbf{I S}_{6}, \uparrow 0\right\rangle\right)=\log \left(\left\langle\mathbf{I S}_{5}, \uparrow 0\right\rangle\right)=\log \left(\left\langle\mathbf{I S}_{4}, \uparrow 0\right\rangle\right)=\log \left(\left\langle\mathbf{I S}_{3}, \uparrow 0\right\rangle\right)=\mathcal{I} \mathcal{S}_{\leq}+p \vee \sim \nabla p$.

Proof. (i). For the equalities $\log \left(\left\langle\mathbf{I S}_{6},\{\hat{1}\}\right\rangle\right)=\log \left(\left\langle\mathbf{I S}_{5},\{\hat{1}\}\right\rangle\right)=\log \left(\left\langle\mathbf{I S}_{4},\{\hat{1}\}\right\rangle\right)=$ $\log \left(\left\langle\mathbf{I S}_{3},\{\hat{1}\}\right\rangle\right)$, it suffices to observe that $\left\langle\mathbf{I S}_{6},\{\hat{1}\}\right\rangle^{*}=\left\langle\mathbf{I S}_{5},\{\hat{1}\}\right\rangle^{*}=\left\langle\mathbf{I S}_{4},\{\hat{1}\}\right\rangle^{*}=\left\langle\mathbf{I S}_{3},\{\hat{1}\}\right\rangle$. Consider $\mathbb{M}=\left\langle\mathbf{I S}_{6}, \uparrow b\right\rangle^{\omega}$ and $\mathbf{R}=\{p \vdash \sim \nabla \sim p\}$. Note for every $v \in \operatorname{Val}_{\mathbb{M} \omega}$ we have that $v(\sim \nabla \sim p) \in D$ iff $v(p)=\{\hat{1}\}^{\omega}$. Hence, from $v \in \operatorname{Val}_{\mathbb{M}^{\omega}}^{\mathrm{R}}$ we have that $v(A) \in D^{\omega}$ iff $v(A)=\{1\}^{\omega}$. As $\mathrm{R}$ is sound in $\left\langle\mathbf{I} \mathbf{S}_{6}, 1\right\rangle^{\omega}$ we obtain the equality $\mathrm{Val}_{\mathbb{M}^{\omega}}^{\mathrm{R}}=\mathrm{Val}_{\left\langle\mathbf{I} \mathbf{S}_{6}, 1\right\rangle^{\omega}}$, thus $\mathcal{I S}_{\leq}+p \vdash \sim \nabla \sim p=\log \left(\left\langle\mathbf{I S}_{6}, 1\right\rangle^{\omega}\right)=\log \left(\left\langle\mathbf{I S}_{6}, 1\right\rangle\right)$.

(ii). That $\log \left(\left\{\left\langle\mathbf{I S}_{3},\{\hat{1}\}\right\rangle,\left\langle\mathbf{I S}_{3}, \uparrow 0\right\rangle\right\}\right)$ is the order-preserving logic of the variety $\mathbb{V}\left(\mathbf{I S}_{3}\right)$ follows from the observation that $\vdash_{\mathbb{V}(\mathrm{K})}^{\leq}=\vdash_{\mathrm{K}}^{<}$holds for any class K. Applying Theorem 5.2 to the multiple-conclusion axiomatization for $\log \left(\left\{\left\langle\mathbf{I S}_{3},\{\hat{1}\}\right\rangle,\left\langle\mathbf{I S}_{3}, \uparrow 0\right\rangle\right\}\right)$ presented in Example 5.17 in the following Section, we conclude that collecting all the $\mathbf{s}_{i}^{\vee}$ for $1 \leq i \leq 21$ provides a single-conclusion axiomatization of $\log \left(\left\{\left\langle\mathbf{I} \mathbf{S}_{3},\{\hat{1}\}\right\rangle,\left\langle\mathbf{I S}_{3}, \uparrow 0\right\rangle\right\}\right)$. The latter equality in the statement follows as the new rules, corresponding to $\mathbf{s}_{15}^{\vee}, \mathbf{s}_{16}^{\vee}$ and $\mathbf{s}_{20}^{\vee}$, are exactly ones that fail in $\left\langle\mathbf{I S}_{6}, \uparrow a\right\rangle$.

(iii). For the equalities $\log \left(\left\langle\mathbf{I S}_{6}, \uparrow 0\right\rangle\right)=\log \left(\left\langle\mathbf{I S}_{5}, \uparrow 0\right\rangle\right)=\log \left(\left\langle\mathbf{I S}_{4}, \uparrow 0\right\rangle\right)=\log \left(\left\langle\mathbf{I S}_{3}, \uparrow\right.\right.$ $0\rangle)$, it suffices to observe that $\left\langle\mathbf{I S}_{6}, \uparrow 0\right\rangle^{*}=\left\langle\mathbf{I S}_{5}, \uparrow 0\right\rangle^{*}=\left\langle\mathbf{I S}_{4}, \uparrow 0\right\rangle^{*}=\left\langle\mathbf{I S}_{3}, \uparrow 0\right\rangle$. Let $\mathrm{R}=\{p \vee \sim \nabla p\}$. In order to obtain the last equality, in the light of Proposition [5.13 (i), it is enough to show that $\mathrm{Val}_{\left\langle\mathbf{I S}_{3}, \uparrow 0\right\rangle}=\mathrm{Val}_{\left\langle\mathbf{I S}_{6}, \uparrow a\right\rangle}^{\mathrm{R}}$. This follows from the fact that given $v \in \operatorname{Val}_{\left\langle\mathbf{I} \mathbf{S}_{6}, \uparrow a\right\rangle}^{\mathrm{R}}$ we must have that for every formula $\varphi$ both $v(\varphi) \neq b$ and $v(\varphi) \neq 0$ (therefore also $v(\varphi) \neq 1$ ), and the fact that $\mathrm{R}$ is sound w.r.t. $\left\langle\mathbf{I S}_{3}, \uparrow 0\right\rangle$.

5.4. Analtytic calculi. Let $\Lambda \subseteq F m$ and let $\mathrm{R}$ be a set of multiple-conclusion rules. We write $3 \triangleright_{\mathrm{R}}^{\Lambda} \Delta$ when there exists an R-proof of $\Delta$ from $\Gamma$ where only formulas in $\Lambda$ occur. Let $\Phi \subseteq F m$. We say that $\mathrm{R}$ is $\Phi$-analytic if when $\Gamma \triangleright_{\mathrm{R}} \Delta$ then $\Gamma \triangleright_{\mathrm{R}}^{\Upsilon_{\Phi}} \Delta$ with $\Upsilon=\operatorname{sub}(\Gamma \cup \Delta)$ and $\Upsilon_{\Phi}=\Upsilon \cup\left\{A^{\sigma}: A \in \Phi, \sigma: P \rightarrow \Upsilon\right\}$. Intuitively, this means that an R-proof of $\Delta$ from $\Gamma$ needs only to use formulas which are subformulas of $\Gamma \cup \Delta$, or instances of $\Phi$ with such subformulas. Hence, formulas in $\Upsilon_{\Phi}$ can be seen as 'generalized subformulas'.

Given $\Phi \subseteq F m$, let $\Phi^{\nabla}:=\Phi \cup\{\nabla p, \sim \nabla p, \nabla \sim p, \sim \nabla \sim p\}$. The Theorem below is a refinement of Theorem 5.3 that applies when we depart from calculus that is analytic, entailing that the operation described in Theorem 5.3 preserves analyticity.

Theorem 5.15. Let $\mathcal{M}$ be a class of $\Sigma$-matrices. If $\mathrm{R}$ is an $\Phi$-analytic axiomatization of $\triangleright_{\widehat{\mathcal{M}}}$ then $\mathrm{R} \cup \mathrm{R}_{\nabla}$ is an $\Phi^{\nabla}$-analytic axiomatization of $\triangleright_{\mathcal{M}} \nabla$.

\footnotetext{
${ }^{3}$ Note that in general $\triangleright_{\mathrm{R}}^{\Lambda}$ is not a multiple-conclusion consequence relation. It still satisfies dilution and cut for set properties, but only weaker versions of overlap and substitution invariance.
} 
Proof. The proof can be easily obtained by adapting the proof of Theorem 5.3. Let $\Upsilon=$ $\operatorname{sub}(\Gamma \cup \Delta)$ and $\Lambda=\Upsilon_{\Phi \nabla}$. Assume $\Gamma \not_{\mathrm{R} \cup \mathrm{R}_{\nabla}}^{\Lambda} \Delta$. Then, by cut for sets, there is a partition $\langle T, F\rangle$ of $\Lambda$ such that $\Gamma \subset T, \Delta \subset F$ and $T \not_{\mathrm{R}_{\mathrm{R}}}^{\Lambda} F$. Since $\mathrm{R} \subseteq \mathrm{R} \cup \mathrm{R}_{\nabla}$, we know that $T \ngtr_{\mathrm{R} \cup \mathrm{R}_{\nabla}}^{\Lambda} F$. Therefore, since $\Upsilon_{\Phi} \subseteq \Upsilon_{\Phi \nabla}$, by $\Phi$-analyticity of $\mathrm{R}$ we have that $T \triangleright_{\widehat{\mathcal{M}}} F$ and we can pick $v \in \operatorname{Hom}_{\Sigma}\left(L_{\Sigma \nabla}, \widehat{\mathbb{M}}\right)$ for some $\mathbb{M} \in \mathcal{M}$ such that $v(T) \subseteq D$ and $v(F) \cap D=\emptyset$. Noting that for every $A \in \Upsilon$ we have $\nabla A, \nabla \sim A, \sim \nabla A, \sim \nabla \sim A \in \Upsilon_{\mathcal{S}_{\nabla}}=\Lambda$, we can define $v^{\prime}: \Upsilon \rightarrow \mathbb{M}^{\nabla}$ as in Theorem [5.3. That $v^{\prime}$ respects all the connectives (and is therefore a partial $\mathbb{M}^{\nabla}$ valuation) follows from the fact that in the proof of Theorem [5.3 we only used instances of the rules using formulas in $\Upsilon$ yielding formulas in $\Upsilon_{\mathcal{S}_{\nabla}}=\Lambda$. As $\mathbb{M}$ is a matrix, $v^{\prime}$ can be extended to a total valuation and therefore $\Gamma \phi_{\mathcal{M} \nabla} \Delta$, thus concluding the proof.

The papers [17, 18] introduced a general method for obtaining analytic calculi for logics given by (partial non-deterministic) matrices whenever a certain expressiveness requirement is met. In particular, for the logic determined by a single matrix $\mathbb{M}=\langle\mathbf{A}, D\rangle$, it suffices that $\mathbb{M}$ be monadic [22, p. 265]. This means that, for all $x, y \in A$ with $x \neq y$, there is a one-variable separating formula, that is, a formula $\varphi(p)$ such that $\varphi(x) \in D$ and $\varphi(y) \notin D$ (or vice versa).

From now on, let us fix the separating set $\mathcal{S}:=\{p, \sim p\}$. Applying the above-described method, we obtain the following axiomatization for $\mathcal{B}$.

Example 5.16. The matrix $\left\langle\mathbf{D M}_{4}, \uparrow a\right\rangle$ is monadic with set of separators $\mathcal{S}$. We can therefore apply the method introduced in [18] to we obtain the following $\mathcal{S}$-analtytic axiomatization for $\mathcal{B}=\log \left\langle\mathbf{D M}_{4}, \uparrow a\right\rangle$ :

$$
\begin{aligned}
& \frac{p}{\sim \sim p} \quad \frac{\sim \sim p}{p} \\
& \frac{p \wedge q}{p} \quad \frac{p \wedge q}{q} \quad \frac{p, q}{p \wedge q} \quad \frac{\sim p}{\sim(p \wedge q)} \quad \frac{\sim q}{\sim(p \wedge q)} \quad \frac{\sim(p \wedge q)}{\sim p, \sim q} \\
& \frac{p}{p \vee q} \quad \frac{q}{p \vee q} \quad \frac{p \vee q}{p, q} \quad \frac{\sim p, \sim q}{\sim(p \vee q)} \quad \frac{\sim(p \vee q)}{\sim p} \quad \frac{\sim(p \vee q)}{\sim q} \\
& \bar{\top} \quad \stackrel{\sim \top}{\sim \perp} \stackrel{\perp}{-}
\end{aligned}
$$

Note that this axiomatization coincides with the one presented in [20, Section 9]. Theorem 5.15 then tells us that we can obtain an $\mathcal{S}_{\nabla}$-analtytic axiomatization of $\mathcal{I} \mathcal{S}_{\leq}$by adding $\mathrm{R}_{\nabla}$ to the above rules.

Example 5.17. In [17, Example 5] we showed that the following rules provide an $\mathcal{S}$ analtytic axiomatization of Kleene's logic of order $\mathcal{K}_{\leq}=\log \left\{\left\langle\mathbf{K}_{3},\{1\}\right\rangle,\left\langle\mathbf{K}_{3}, \uparrow a\right\rangle\right\}$.

$$
\begin{gathered}
\frac{p, q}{p \wedge q} \mathrm{~s}_{1} \frac{p \wedge q}{p} \mathrm{~s}_{2} \quad \frac{p \wedge q}{q} \mathrm{~s}_{3} \frac{\sim p}{\sim(p \wedge q)} \mathrm{s}_{4} \frac{\sim q}{\sim(p \wedge q)} \mathrm{s}_{5} \frac{\sim(p \wedge q)}{\sim p, \sim q} \mathrm{~s}_{6} \\
\frac{p}{p \vee q} \mathrm{~s}_{7} \frac{q}{p \vee q} \mathrm{~s}_{8} \frac{\sim(p \vee q)}{\sim p} \mathrm{~s}_{9} \frac{\sim(p \vee q)}{\sim q} \mathrm{~s}_{10} \frac{\sim p, \sim q}{\sim(p \vee q)} \mathrm{s}_{11} \frac{p \vee q}{p, q} \mathrm{~s}_{12} \\
\frac{p}{\sim \sim p} \mathrm{~s}_{13} \frac{\sim \sim p}{p} \mathrm{~s}_{14} \frac{p, \sim p}{q, \sim q} \mathrm{~s}_{15}
\end{gathered}
$$


Since $\left\{\left\langle\mathbf{I S}_{5}, \uparrow 1\right\rangle,\left\langle\mathbf{I S}_{5}, \uparrow a\right\rangle\right\}=\left\{\left\langle\mathbf{K}_{3},\{1\}\right\rangle,\left\langle\mathbf{K}_{3}, \uparrow a\right\rangle\right\}^{\nabla}$, by Theorem 5.15, we have that adding $\mathrm{R}_{\nabla}$ to the above rules gives us a $\mathcal{S}_{\nabla}$-analtytic axiomatization of $\log \left\{\left\langle\mathbf{I} \mathbf{S}_{5}, \uparrow 1\right\rangle,\left\langle\mathbf{I S}_{5}, \uparrow\right.\right.$ a) $\}$.

The method of [18] can also be applied directly to obtain an $\mathcal{S}$-analtytic axiomatization of $\vdash_{\mathbb{V}\left(\mathbf{I S}_{3}\right)}^{\leq}=\log \left\{\left\langle\mathbf{I S}_{3},\{\hat{1}\}\right\rangle,\left\langle\mathbf{I S}_{3}, \uparrow 0\right\rangle\right\}$. Indeed, the $\nabla$-free fragment of $\log \left\{\left\langle\mathbf{I S}_{3},\{\hat{1}\}\right\rangle,\left\langle\mathbf{I S}_{3}, \uparrow\right.\right.$ $a\rangle\}$ is $\log \left\{\left\langle\mathbf{K}_{3},\{1\}\right\rangle,\left\langle\mathbf{K}_{3}, \uparrow a\right\rangle\right\}$. The latter set of matrices can be viewed as a partial matrix [18, Section 2.2] and is monadic with separating set $\mathcal{S}$ (in which $\nabla$ does not occur). Therefore, the modularity of the method of [18] tells us we just have to add the rules corresponding to $\nabla$. Hence, it suffices to add the rules:

$$
\frac{\sim p, \nabla p}{p} \mathrm{~s}_{16} \quad \frac{\sim p}{p, \sim \nabla p} \mathrm{~s}_{17} \quad \frac{p}{\nabla p} \mathrm{~s}_{18} \quad \underline{p, \sim \nabla p} \mathrm{~s}_{19} \quad \frac{\sim \nabla p}{\sim p} \mathrm{~s}_{20} \quad \frac{}{\sim p, \nabla p} \mathrm{~s}_{21}
$$

which give us the single-conclusion axiomatization relative to $\mathcal{I S}_{\leq}$mentioned in Theorem 5.14 (ii).

\section{Conclusions And future WORK}

As we have shown, the lattice of super-Belnap logics is embeddable in the lattice of extensions of $\mathcal{I S} \mathcal{S}_{<}$. This connection provides significant insight, but it also suggests that fully describing the latter is at least as complex as describing the former, whose structure is still not completely understood (see [1]). Obviously, in the present study we have only scratched the surface of the general problem. A reasonable starting point for a systematic account of the extensions of $\mathcal{I} \mathcal{S}_{\leq}$is to adapt the various results and strategies in [21, 1, 20] to the richer setting of involutive Stone algebras. We mention, in particular, the issues of characterizing the reduced models of extensions of $\mathcal{I} \mathcal{S}_{\leq}$, and that of providing a general semantical description of the explosive extensions (in the sense of [1, 20]) of logics over $\mathcal{I} \mathcal{S}_{\leq}$.

An altogether different perspective on extensions of $\mathcal{I} \mathcal{S}_{<}$, which has not been considered in the present paper, comes from the observation made in [7, Sec. 6] that $\mathcal{I} \mathcal{S}_{\leq}$may be viewed as a paraconsistent logic, more precisely as a Logic of Formal Inconsistency (LFIs) in the sense of $\mathrm{N}$. da Costa 12 . Indeed, $\mathcal{I} \mathcal{S}_{\leq}$(and so its extensions) can be equivalently presented in a language that replaces the $\nabla$ connective with either the consistency operator (o) or the inconsistency operator $(\bullet)$ that are usually considered in the literature on LFIs. One possible definition is $\nabla \varphi:=\sim \circ \varphi \vee \varphi$, and, conversely, one may define $\circ \varphi:=\sim \nabla(\varphi \wedge \sim \varphi)$ and $\bullet \varphi:=\nabla(\varphi \wedge \sim \varphi)$.

From a philosophical logic point of view, the advantage of the latter presentation is that the operators $\circ$ and $\bullet$ have a clearer logical interpretation than $\nabla$, namely, $\circ \varphi$ means ' $\varphi$ is consistent' and $\bullet \varphi$ means ' $\varphi$ is inconsistent'; on the other hand, $\nabla$ behaves very well from the points of view of algebraic logic and duality theory, for it satisfies the usual axioms for modal operators. A more interesting observation is that, in the setting of LFIs, the (in)consistency operators are usually required to satisfy much weaker axioms than those that result from the definitions $\circ \varphi:=\sim \nabla(\varphi \wedge \sim \varphi)$ and $\bullet \varphi:=\nabla(\varphi \wedge \sim \varphi)$ within $\mathcal{I S}_{\leq}$. This suggests a potentially fruitful project for future research: namely, a systematic study of more general algebraic structures (e.g. De Morgan algebras endowed with a consistency operator) corresponding to weaker logics (viewed as LFIs) that approximate $\mathcal{I S}_{\leq}$from below. 


\section{COMPLiAnCE WITH ETHICAL STANDARDS}

Funding: Research funded by FCT/MCTES through national funds and when applicable co-funded by EU under the project UIDB/EEA/50008/2020 and by the Conselho Nacional de Desenvolvimento Científico e Tecnológico (CNPq, Brazil), under the grant 313643/2017-2 (Bolsas de Produtividade em Pesquisa - PQ).

Conflict of Interest: The authors declare that they have no conflict of interest.

Ethical approval: This article does not contain any studies with human participants or animals.

\section{REFERENCES}

[1] H. Albuquerque, A. Prenosil and U. Rivieccio. An algebraic view of super-Belnap logics. Studia Logica, 105(6):1051-1086, 2017.

[2] N. D. Belnap, Jr. A useful four-valued logic. In J. M. Dunn and G. Epstein, editors, Modern uses of multiple-valued logic (Fifth Internat. Sympos., Indiana Univ., Bloomington, Ind., 1975), pages 5-37. Episteme, Vol. 2. Reidel, Dordrecht, 1977.

[3] S. Burris and H. P. Sankappanavar. A course in Universal Algebra. The Millennium edition, 2000.

[4] C. Caleiro, S. Marcelino, and J. Marcos. Combining fragments of classical logic: When are interaction principles needed? Soft Computing, 23(7): 2213-2231, 2019

[5] C. Caleiro. and S. Marcelino. On axioms and rexpansions. Outstanding Contributions to Logic (in print)

[6] L. Cantú. Sobre la lógica que preserva grados de verdad asociada a las álgebras de Stone involutivas. Masters dissertation, Universidad Nacional del Sur (Bahía Blanca, Argentina), 2019.

[7] L. Cantú and M. Figallo. On the logic that preserves degrees of truth associated to involutive Stone algebras. Logic Journal of the IGPL, 2018, doi: 10.1093/jigpal/jzy071.

[8] R. Cignoli and M. S. de Gallego. The lattice structure of some Łukasiewicz algebras. Algebra Universalis, 13(3):315-328, 1981.

[9] R. Cignoli and M. S. de Gallego. Dualities for some De Morgan algebras with operators and Lukasiewicz algebras. Journal of the Australian Mathematical Society, 34(3):377-393, 1983.

[10] R. Cignoli, I. M. L. D'Ottaviano, and D. Mundici. Algebraic foundations of many-valued reasoning, volume 7 of Trends in Logic-Studia Logica Library. Kluwer Academic Publishers, Dordrecht, 2000.

[11] D. M. Clark and B. A. Davey. Natural dualities for the working algebraist, volume 57 of Cambridge Studies in Advanced Mathematics. Cambridge University Press, Cambridge, 1998.

[12] N.C.A. da Costa. Calculs propositionnels pour les systemes formels inconsistants. Compte Rendu Acad. des Sciences (Paris), 257(3):3790-3793, 1963.

[13] B. A. Davey and H. A. Priestley. Introduction to lattices and order. Cambridge University Press, Cambridge, 1990.

[14] J. M. Font. Belnap's four-valued logic and De Morgan lattices. Logic Journal of the I.G.P.L., 5(3):413440, 1997.

[15] J. M. Font. Abstract algebraic logic: An introductory textbook. College Publications, 2016.

[16] J. M. Font and R. Jansana. A general algebraic semantics for sentential logics, volume 7 of Lecture Notes in Logic. Springer-Verlag, second edition, 2009.

[17] S. Marcelino and C. Caleiro. Analytic calculi for monadic PNmatrices. In International Workshop on Logic, Language, Information, and Computation (WoLLIC 2019), pp. 84-98. Springer, Berlin, Heidelberg, 2019.

[18] S. Marcelino and C. Caleiro. Axiomatizing non-deterministic many-valued generalized consequence relations. Synthese (2019), doi: 10.1007/s11229-019-02142-8.

[19] A. Pietz and U. Rivieccio. Nothing but the truth. Journal of Philosophical Logic, 42(1):125-135, 2013.

[20] A. Prenosil. The lattice of super-Belnap logics. Submitted.

[21] U. Rivieccio. An infinity of super-belnap logics. Journal of Applied Non-Classical Logics, 22(4):319-335, 2012.

[22] D. J. Shoesmith and T. J. Smiley. Multiple-conclusion logic. Cambridge University Press, Cambridge, 1978.

[23] R. Wójcicki. Theory of logical calculi. Basic theory of consequence operations, volume 199 of Synthese Library. Reidel, Dordrecht, 1988. 